\title{
38. SEISMIC VELOCITIES OF DIABASE AND BASALT FROM MIDDLE VALLEY SILLS AND FLOWS, NORTHERN JUAN DE FUCA RIDGE ${ }^{1}$
}

\author{
Henrike M. Gröschel-Becker, ${ }^{2}$ Heinrich W. Villinger, ${ }^{3}$ Boris A. Konyukhov,,${ }^{4}$ Gerardo J. Iturrino, ${ }^{2}$ and Nikolas I. Christensen ${ }^{5}$
}

\begin{abstract}
The physical structure of intercalated sills and indurated sediment forming the shallow basement at hydrothermally active sedimented spreading centers was not well known prior to Ocean Drilling Program Leg 139 drilling in Middle Valley. Compressional- and shear-wave velocities for 28 igneous rock samples from Holes $856 \mathrm{~A}, 857 \mathrm{C}, 857 \mathrm{D}$, and $858 \mathrm{G}$ were measured in the laboratory at elevated confining pressures in a study of the acoustic character of the diabasic sills and a basalt flow. These data are useful in interpretation of seismic reflection profiles and construction of synthetic seismograms (Rohr and Gröschel-Becker et al., 1992 and this volume). The study focused on the 24 diabase samples from Site 857 that represent a continuous section from 471.1 mbsf, the first sill below the sediment cap, to $936.2 \mathrm{mbsf}$. The mean $V_{p}$ of the 28 samples is $5.65 \mathrm{~km} / \mathrm{s}$ and $5.77 \mathrm{~km} / \mathrm{s}$ at 50 and 100 $\mathrm{MPa}$, respectively. Mean $V_{s}$ values are $3.07 \mathrm{~km} / \mathrm{s}$ at $50 \mathrm{MPa}$ and $3.16 \mathrm{~km} / \mathrm{s}$ at $100 \mathrm{MPa}$. The mean bulk density at atmospheric pressure is $2.81 \mathrm{~g} / \mathrm{cm}^{3}$. These data agree with values measured for relatively fresh, unweathered mid-ocean ridge basalts. The results suggest that different emplacement and alteration histories can produce mineral assemblages with similar physical properties, a consideration in the interpretation of remotely sensed data. Porosities for Site 857 diabases are unusually high compared to a reported upper Layer 2 average porosity of $2 \%$; they range from $3.95 \%$ to $14.38 \%$ with a mean of $7.2 \%$. Highly altered sill margins at Site 857 are characterized by low $V_{p}$, low densities, and high porosities, whereas less altered sill interiors have high $V_{p}$, high densities, and lower porosities. Drilling-related microcracks and partially filled cracks, veins, and intergranular pores inherent to the rocks contribute to the high porosities. Velocities, densities, and porosities are highly variable owing more to compositional and textural changes associated with varying degrees of hydrothermal alteration and the presence of open and filled veins, cracks, and vugs than to primary mineralogy. These factors explain the nonsystematic velocity differences between adjacent oriented minicores. Thin-section observations showed that the variations cannot be interpreted in terms of $V_{p}$ anisotropy resulting from preferred mineral orientations or oriented microcrack populations. Higher vertical velocities measured in shipboard cube samples may have resulted from the opening of pre-existing subvertical microcracks at laboratory pressures. Shore-based computer-assisted image analyses of Site 857 thin sections quantified percentages of minerals and physical discontinuities that influence the physical properties measured in the laboratory. $V_{p}$ and densities can be correlated with the percentages of oxide and sulfide minerals. No correlation between velocity and percentage of secondary minerals is observed, probably because some secondary minerals reduce velocities and densities while others increase them.
\end{abstract}

\section{INTRODUCTION}

Compressional- and shear-wave velocities $\left(V_{p}\right.$ and $\left.V_{s}\right)$ of oceanic igneous rocks, measured in the laboratory at various confining pressures using techniques similar to the pulse-transmission method of Birch $(1960,1961)$, are used to interpret the seismic properties of oceanic crust in terms of density, porosity, and rock composition. Previous investigations have shown that $V_{p}$ of Layer 2 basalts at in-situ confining pressures are highly variable, ranging from about 3.5 to $6.5 \mathrm{~km} / \mathrm{s}$. Velocities of the uppermost igneous crust measured for basalts by seismic refraction experiments are systematically lower, typically ranging from 2.5 to $5 \mathrm{~km} / \mathrm{s}$ (Christensen, 1972; Fox et al., 1973; Hyndman and Drury, 1976; Christensen et al., 1979; Spudich and Orcutt, 1980; Wilkens et al., 1983; Stephen, 1985; Purdy, 1987). Deep-sea sampling (Hyndman and Drury, 1976; Anderson et al., 1982; Alt et al., 1986) and submersible observations (Rona et al., 1984; Karson and Rona, 1990; Rona et al., 1990) reveal that shallow volcanics are often rubbly, fractured, and brecciated, and characterized by high porosities and numerous alteration minerals. The rapid increase in velocity with depth is attributed to decreasing porosity, which has been shown to control

\footnotetext{
${ }^{1}$ Mottl, M.J., Davis, E.E., Fisher, A.T., and Slack, J.F. (Eds.), 1994. Proc, ODP, Sci. Results, 139: College Station, TX (Ocean Drilling Program).

${ }^{2}$ Rosenstiel School of Marine and Atmospheric Science, University of Miami, 4600 Rickenbacker Causeway, Miami, FL 33149-1098, U.S.A.

${ }^{3}$ Fachbereich Geowissenschaften, Postfach 330 440, Universität Bremen, D-2800 Bremen 33, Federal Republic of Germany.

${ }^{4}$ Pacific Oceanological Institute, U.S.S.R. Academy of Sciences, 7 Radio Street, Vladivostok 690032, Russia

${ }^{5}$ Rock Physics Laboratory, Department of Earth and Atmospheric Sciences, Purdue University, West Lafayette, IN 47907 , U.S.A.
}

velocity and density systematics in shallow oceanic rocks (Becker et al., 1989; Carlson and Herrick, 1990; Wilkens et al., 1991).

Models of oceanic crustal structure are mostly based on geophysical experiments and samples recovered from the thinly-sedimented flanks of mid-ocean ridges, and less frequently on zero-age crust due to sampling difficulties. However, the detailed physical structure of noncontinuous shallow basement at hydrothermally active spreading centers covered by a thick veneer of sediment was not known until Ocean Drilling Program (ODP) Leg 139 drilled in Middle Valley of the northern Juan de Fuca Ridge. Discrete sills or flows of mafic rocks interbedded with altered, indurated sediment were penetrated; these sequences are inferred to grade into purely oceanic basement rocks at depth (Davis and Villinger, 1992).

The goal of this study is to characterize the acoustic structure of the igneous portion of the "layer-cake" basement that deep-sea drilling results from Leg 139 and previous cruises to the Guaymas and Yamato Basins (Einsele et al., 1980; Curray, Moore, et al., 1982; Lonsdale and Becker, 1985; Ingle et al., 1990; Tamaki, Pisciotto, Allan, et al., 1990; Tamaki et al., 1992) indicate is typical for spreading centers near sources of copious sediment supply (Langseth et al., 1992). Samples were obtained from an uplifted, sediment-covered hill near a massive sulfide outcrop at Site 856 , and from two sites, $1.6 \mathrm{~km}$ apart, located above a buried basement block within a high heat flow region that parallels the rift. Site 857 penetrated about $500 \mathrm{~m}$ of sediment that overlies a hydrothermal reservoir containing high-temperature fluids and Site 858 was drilled into a region of active venting associated with a small basement edifice. The effects of porosity, density, mineralogy, and alteration on the sills' velocity structure (Tables 1 and 2) will be evaluated in the next sections, with emphasis on results from the 24 Site 857 samples. Our results are compared to physical properties data 
Table 1. Variations in directional compressional- and shear-wave velocities, and compositional descriptions for samples from Holes 856 A, 857C, 857D, and 858G measured to $100 \mathrm{MPa}$ at the RSMAS Petrophysics Laboratory.

\begin{tabular}{|c|c|c|c|c|c|c|c|c|c|c|c|c|c|}
\hline \multirow[b]{2}{*}{ Sample Description } & \multirow[b]{2}{*}{ Direction } & \multirow{2}{*}{$\begin{array}{l}\text { Velocity } \\
(\mathrm{km} / \mathrm{s})\end{array}$} & \multicolumn{10}{|c|}{ Pressure (MPa) } & \multirow[b]{2}{*}{ Comments } \\
\hline & & & 10 & 20 & 30 & 40 & 50 & 60 & 70 & 80 & 90 & 100 & \\
\hline $\begin{array}{l}\text { 139-856A-14X-1, } 61-63 \mathrm{~cm} \\
\quad \rho=2.89 \mathrm{~g} / \mathrm{cc} \\
\phi=1.8 \% \\
\text { Medium-grained porphyritic diabase }\end{array}$ & $\mathrm{h}$ & $\begin{array}{l}V_{p} \\
V_{s l} \\
V_{s 2}\end{array}$ & $\begin{array}{l}6.10 \\
3.29 \\
3.29\end{array}$ & $\begin{array}{l}6.20 \\
3.31 \\
3.31\end{array}$ & $\begin{array}{l}6.27 \\
3.22 \\
3.22\end{array}$ & $\begin{array}{l}6.31 \\
3.35 \\
3.33\end{array}$ & $\begin{array}{l}6.34 \\
3.37 \\
3.35\end{array}$ & $\begin{array}{l}6.36 \\
3.40 \\
3.37\end{array}$ & $\begin{array}{l}6.38 \\
3.42 \\
3.40\end{array}$ & $\begin{array}{l}6.41 \\
3.41 \\
3.42\end{array}$ & $\begin{array}{l}6.43 \\
3.44 \\
3.42\end{array}$ & $\begin{array}{l}6.45 \\
3.44 \\
3.43\end{array}$ & $\begin{array}{l}\text { Unit I; Piece } 4 \text {. Pheno } 4 \% \text { ol, } 7 \% \\
\text { plag, } 1 \% \text { cpx, } 2 \% \text { spinel. GM } 36 \% \\
\text { cpx, } 43 \% \text { plag, } 1 \% \text { mag. } 0.5 \% \\
\text { random veins. SM } 3 \% \text { clay, } 1 \% \text { chl } \\
1 \% \text { talc, } 1 \% \text { Po (?). }\end{array}$ \\
\hline $\begin{array}{l}\text { 139-856A-14X-1, } 66-68 \mathrm{~cm} \\
\quad \rho=2.92 \mathrm{~g} / \mathrm{cc} \\
\quad \phi=2.3 \% \\
\text { Medium-grained porphyritic diabase }\end{array}$ & v & $\begin{array}{l}V_{p} \\
V_{s l} \\
V_{s 2}\end{array}$ & $\begin{array}{l}7.09 \\
3.65 \\
3.63\end{array}$ & $\begin{array}{l}7.13 \\
3.66 \\
3.64\end{array}$ & $\begin{array}{l}7.17 \\
3.67 \\
3.66\end{array}$ & $\begin{array}{l}7.20 \\
3.68 \\
3.66\end{array}$ & $\begin{array}{l}7.22 \\
3.68 \\
3.68\end{array}$ & $\begin{array}{l}7.24 \\
3.70 \\
3.69\end{array}$ & $\begin{array}{l}7.26 \\
3.73 \\
3.72\end{array}$ & $\begin{array}{l}7.29 \\
3.74 \\
3.74\end{array}$ & $\begin{array}{l}7.31 \\
3.74 \\
3.74\end{array}$ & $\begin{array}{l}7.33 \\
3.76 \\
3.75\end{array}$ & $\begin{array}{l}\text { Unit } 1 \text {; Piece } 5 \text {. As above, with } 1 \% \\
\text { random talc-coated fractures. }\end{array}$ \\
\hline $\begin{array}{l}139-857 \mathrm{C}-64 \mathrm{R}-1,21-23 \mathrm{~cm} \\
\quad \rho=2.77 \mathrm{~g} / \mathrm{cc} \\
\quad \begin{array}{l}\quad=10.7 \% \\
\text { Coarse-grained diabase }\end{array}\end{array}$ & h & $\begin{array}{l}V_{p} \\
V_{s l} \\
V_{s 2}\end{array}$ & $\begin{array}{l}4.95 \\
2.69 \\
2.69\end{array}$ & $\begin{array}{l}5.04 \\
2.74 \\
2.72\end{array}$ & $\begin{array}{l}5.10 \\
2.78 \\
2.78\end{array}$ & $\begin{array}{l}5.13 \\
2.81 \\
2.80\end{array}$ & $\begin{array}{l}5.16 \\
2.84 \\
2.83\end{array}$ & $\begin{array}{l}5.18 \\
2.87 \\
2.85\end{array}$ & $\begin{array}{l}5.20 \\
2.91 \\
2.87\end{array}$ & $\begin{array}{l}5.23 \\
2.92 \\
2.87\end{array}$ & $\begin{array}{l}5.24 \\
2.93 \\
2.88\end{array}$ & $\begin{array}{l}5.25 \\
2.93 \\
2.88\end{array}$ & $\begin{array}{l}\text { Unit } 8 \text {; Piece } 2.42 \% \text { plag, } 38 \% \\
\text { cpx, } 4 \% \text { ilm. SM } 5 \% \text { clay, } 8 \% \text { chl, } \\
1 \% \text { epi, } 2 \% \text { py/cpy. }\end{array}$ \\
\hline $\begin{array}{l}139-857 \mathrm{C}-64 \mathrm{R}-1,24-27 \mathrm{~cm} \\
\quad \rho=2.74 \mathrm{~g} / \mathrm{cc} \\
\quad \begin{array}{l}\quad=9.7 \% \\
\text { Coarse-grained diabase }\end{array}\end{array}$ & $\mathrm{v}$ & $\begin{array}{l}V_{p} \\
V_{s I} \\
V_{s 2}\end{array}$ & $\begin{array}{l}5.61 \\
2.86 \\
2.84\end{array}$ & $\begin{array}{l}5.76 \\
3.02 \\
2.95\end{array}$ & $\begin{array}{l}5.88 \\
3.04 \\
2.97\end{array}$ & $\begin{array}{l}5.96 \\
3.05 \\
2.99\end{array}$ & $\begin{array}{l}6.01 \\
3.06 \\
3.00\end{array}$ & $\begin{array}{l}6.04 \\
3.08 \\
3.04\end{array}$ & $\begin{array}{l}6.07 \\
3.11 \\
3.07\end{array}$ & $\begin{array}{l}6.09 \\
3.12 \\
3.08\end{array}$ & $\begin{array}{l}6.10 \\
3.12 \\
3.09\end{array}$ & $\begin{array}{l}6.13^{*} \\
3.12 \\
3.10\end{array}$ & $\begin{array}{l}\text { Unit } 8 \text {; Piece } 2 \text {. More py as } \mathrm{xl} \\
\text { aggregates and along minor cracks. }\end{array}$ \\
\hline $\begin{array}{l}\text { 139-857D-IR-1, 24-26 cm } \\
\qquad \begin{array}{l}\rho \\
=2.58 \mathrm{~g} / \mathrm{cc}\end{array} \\
\quad=14.4 \% \\
\text { Fine-grained microporphyritic diabase }\end{array}$ & h & $\begin{array}{l}V_{p} \\
V_{s l} \\
V_{s 2}\end{array}$ & $\begin{array}{l}4.11 \\
2.18 \\
2.13\end{array}$ & $\begin{array}{l}4.17 \\
2.21 \\
2.16\end{array}$ & $\begin{array}{l}4.23 \\
2.23 \\
2.18\end{array}$ & $\begin{array}{l}4.26 \\
2.25 \\
2.19\end{array}$ & $\begin{array}{l}4.29 \\
2.26 \\
2.20\end{array}$ & $\begin{array}{l}4.33 \\
2.27 \\
2.22\end{array}$ & $\begin{array}{l}4.35 \\
2.28 \\
2.23\end{array}$ & $\begin{array}{l}4.37 \\
2.29 \\
2.23\end{array}$ & $\begin{array}{l}4.39 \\
2.29 \\
2.23\end{array}$ & $\begin{array}{l}4.40 \\
2.29 \\
2.24\end{array}$ & $\begin{array}{l}\text { Unit } 13 \text {; Piece } 4 \text {; below sedi- } \\
\text { ment contact. } 30 \% \text { (?) plag. SM } \\
30 \% \text { clay, } 30 \% \text { chl, } 10 \% \text { qtz. } \\
4-5 \% \text { random chl-filled veinlets. }\end{array}$ \\
\hline $\begin{array}{l}139-857 \mathrm{D}-1 \mathrm{R}-1,28-31 \mathrm{~cm} \\
\qquad \begin{array}{l}\rho \\
\quad=2.56 \mathrm{~g} / \mathrm{cc}\end{array} \\
\quad \begin{array}{l}=12.9 \% \\
\text { Fine-grained microporphyritic diabase }\end{array}\end{array}$ & $\mathrm{v}$ & $\begin{array}{l}V_{p} \\
V_{s l} \\
V_{s 2}\end{array}$ & $\begin{array}{l}4.13 \\
2.20 \\
2.17\end{array}$ & $\begin{array}{l}4.26 \\
2.24 \\
2.22\end{array}$ & $\begin{array}{l}4.32 \\
2.29 \\
2.26\end{array}$ & $\begin{array}{l}4.35 \\
2.30 \\
2.27\end{array}$ & $\begin{array}{l}4.38 \\
2.31 \\
2.28\end{array}$ & $\begin{array}{l}4.40 \\
2.32 \\
2.29\end{array}$ & $\begin{array}{l}4.42 \\
2.33 \\
2.31\end{array}$ & $\begin{array}{l}4.42 \\
2.33 \\
2.32\end{array}$ & $\begin{array}{l}4.44 \\
2.34 \\
2.32\end{array}$ & $\begin{array}{l}4.46 \\
2.34 \\
2.32\end{array}$ & $\begin{array}{l}\text { Unit } 13 \text {; Piece } 4 \text {. Finer, fewer } \\
\text { veins with some assoc. sulfide. }\end{array}$ \\
\hline $\begin{array}{l}139-857 \mathrm{D}-4 \mathrm{R}-1,66-68 \mathrm{~cm} \\
\quad \rho=2.80 \mathrm{~g} / \mathrm{cc} \\
\quad \begin{array}{l}\quad=6.5 \% \\
\text { Coarse-grained metadiabase }\end{array}\end{array}$ & $\mathrm{h}$ & $\begin{array}{l}V_{p} \\
V_{s 1} \\
V_{s 2}\end{array}$ & $\begin{array}{l}5.50 \\
2.81 \\
2.84\end{array}$ & $\begin{array}{l}5.67 \\
2.91 \\
2.95\end{array}$ & $\begin{array}{l}5.77 \\
2.96 \\
2.99\end{array}$ & $\begin{array}{l}5.80 \\
2.99 \\
3.05\end{array}$ & $\begin{array}{l}5.84 \\
3.03 \\
3.04\end{array}$ & $\begin{array}{l}5.87 \\
3.06 \\
3.05\end{array}$ & $\begin{array}{l}5.89 \\
3.10 \\
3.08\end{array}$ & $\begin{array}{l}5.90 \\
3.11 \\
3.09\end{array}$ & $\begin{array}{l}5.92 \\
3.12 \\
3.09\end{array}$ & $\begin{array}{c}5.93^{*} \\
* * * * \\
* * *\end{array}$ & $\begin{array}{l}\text { Unit } 15 ; \text { Piece } 9.35 \% \text { plag, } 25 \% \\
\text { cpx, } 5 \% \text { ilm/mag. SM } 20 \% \text { chl, } \\
5 \% \text { epi, } 5 \% \text { act, } 5 \% \text { py/po/cpy in } \\
\text { large aggregates. }\end{array}$ \\
\hline $\begin{array}{l}139-857 \mathrm{D}-4 \mathrm{R}-1,69-72 \mathrm{~cm} \\
\quad \rho=2.82 \mathrm{~g} / \mathrm{cc} \\
\quad \begin{array}{l}\phi=6.2 \% \\
\text { Coarse-grained metadiabase }\end{array}\end{array}$ & $\mathrm{v}$ & $\begin{array}{l}V_{p} \\
V_{s l} \\
V_{s 2}\end{array}$ & $\begin{array}{l}5.19 \\
2.61 \\
2.61\end{array}$ & $\begin{array}{l}5.40 \\
2.80 \\
2.78\end{array}$ & $\begin{array}{l}5.51 \\
2.89 \\
2.89\end{array}$ & $\begin{array}{l}5.59 \\
2.95 \\
2.95\end{array}$ & $\begin{array}{l}5.66 \\
3.00 \\
2.98\end{array}$ & $\begin{array}{l}5.71 \\
3.04 \\
3.01\end{array}$ & $\begin{array}{l}5.75 \\
3.09 \\
3.05\end{array}$ & $\begin{array}{l}5.76 \\
3.10 \\
3.07\end{array}$ & $\begin{array}{l}5.79 \\
3.12 \\
3.08\end{array}$ & $\begin{array}{l}5.81 \\
3.13 \\
3.10\end{array}$ & Unit 15; Piece 9. As above. \\
\hline $\begin{array}{l}139-857 \mathrm{D}-9 \mathrm{R}-1,51-53 \mathrm{~cm} \\
\qquad \begin{array}{l}\rho \\
\quad=2.87 \mathrm{~g} / \mathrm{cc}\end{array} \\
\text { Fine-grained porphyritic diabase }\end{array}$ & h & $\begin{array}{l}V_{p} \\
V_{s l}^{p} \\
V_{s 2}\end{array}$ & $\begin{array}{l}5.32 \\
2.95 \\
2.95\end{array}$ & $\begin{array}{l}5.41 \\
3.00 \\
2.99\end{array}$ & $\begin{array}{l}5.47 \\
3.03 \\
3.02\end{array}$ & $\begin{array}{l}5.50 \\
3.07 \\
3.05\end{array}$ & $\begin{array}{l}5.54 \\
3.09 \\
3.07\end{array}$ & $\begin{array}{l}5.56 \\
3.12 \\
3.09\end{array}$ & $\begin{array}{l}5.58 \\
3.15 \\
3.12\end{array}$ & $\begin{array}{l}5.59 \\
3.16 \\
3.12\end{array}$ & $\begin{array}{l}5.61 \\
3.18 \\
3.13\end{array}$ & $\begin{array}{l}5.63 \\
3.16 \\
3.13\end{array}$ & $\begin{array}{l}\text { Unit 16C; Piece 9. Pheno } 0.5 \% \\
\text { cpx. GM } 42 \% \text { plag, } 40 \% \text { cpx, } 5 \% \\
\text { ilm/mag. SM } 5 \% \text { chl, } 2 \% \text { dissem. } \\
\text { py. Minor chl-filled vein. }\end{array}$ \\
\hline $\begin{array}{l}139-857 \mathrm{D}-9 \mathrm{R}-1,54-57 \mathrm{~cm} \\
\qquad \begin{array}{l}\rho \\
\phi\end{array}=5.88 \mathrm{~g} / \mathrm{cc} \\
\text { Fine-grained porphyritic diabase }\end{array}$ & $\mathrm{v}$ & $\begin{array}{l}V_{p} \\
V_{s l} \\
V_{s 2}\end{array}$ & $\begin{array}{l}5.30 \\
3.06 \\
3.10\end{array}$ & $\begin{array}{l}5.39 \\
3.07 \\
3.03\end{array}$ & $\begin{array}{l}5.47 \\
3.08 \\
3.06\end{array}$ & $\begin{array}{l}5.53 \\
3.11 \\
3.08\end{array}$ & $\begin{array}{l}5.59 \\
3.13 \\
3.10\end{array}$ & $\begin{array}{l}5.61 \\
3.16 \\
3.13\end{array}$ & $\begin{array}{l}5.67 \\
3.20 \\
3.17\end{array}$ & $\begin{array}{l}5.69 \\
3.20 \\
3.18\end{array}$ & $\begin{array}{l}5.71 \\
3.22 \\
3.18\end{array}$ & $\begin{array}{l}5.73 \\
3.23 \\
3.19\end{array}$ & $\begin{array}{l}\text { Unit } 16 \mathrm{C} \text {; Piece } 10 \text {. Pheno } 1 \% \mathrm{cpx} \text {. } \\
\text { Minor dissem. sulfide; py/po, qtz } \\
\text { partly fill major crack. }\end{array}$ \\
\hline 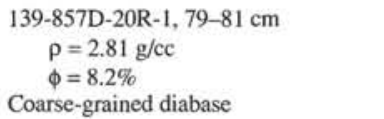 & $\mathrm{h}$ & $\begin{array}{l}V_{p} \\
V_{s l} \\
V_{s 2}\end{array}$ & $\begin{array}{l}5.35 \\
2.68 \\
2.66\end{array}$ & $\begin{array}{l}5.40 \\
2.82 \\
2.76\end{array}$ & $\begin{array}{l}5.44 \\
2.93 \\
2.84\end{array}$ & $\begin{array}{l}5.47 \\
2.96 \\
2.88\end{array}$ & $\begin{array}{l}5.50 \\
2.99 \\
2.91\end{array}$ & $\begin{array}{l}5.52 \\
3.01 \\
2.94\end{array}$ & $\begin{array}{l}5.55 \\
3.04 \\
2.99\end{array}$ & $\begin{array}{l}5.57 \\
3.04 \\
3.00\end{array}$ & $\begin{array}{l}5.59 \\
3.05 \\
3.01\end{array}$ & $\begin{array}{l}5.61 \\
3.05 \\
3.01\end{array}$ & $\begin{array}{l}\text { Unit 19; Piece } 10.39 \% \text { plag, } 30 \% \\
\text { cpx, } 2 \% \text { oxide. SM } 10 \% \text { chl, } 5 \% \\
\text { epi, } 10 \% \text { act, } 3 \% \text { prehnite, } 6 \% \text { py } \\
\text { mostly in large aggregate. }\end{array}$ \\
\hline
\end{tabular}


Table 1 (continued).

\begin{tabular}{|c|c|c|c|c|c|c|c|c|c|c|c|c|c|}
\hline \multirow[b]{2}{*}{ Sample Description } & \multirow[b]{2}{*}{ Direction } & \multirow{2}{*}{$\begin{array}{l}\text { Velocity } \\
(\mathrm{km} / \mathrm{s})\end{array}$} & \multicolumn{10}{|c|}{ Pressure (MPa) } & \multirow[b]{2}{*}{ Comments } \\
\hline & & & 10 & 20 & 30 & 40 & 50 & 60 & 70 & 80 & 90 & 100 & \\
\hline $\begin{array}{l}\text { 139-857D-20R-1, } 83-85 \mathrm{~cm} \\
\rho=2.81 \mathrm{~g} / \mathrm{cc} \\
\quad=8.4 \% \\
\text { Coarse-grained diabase }\end{array}$ & $v$ & $\begin{array}{l}V_{p} \\
V_{s l} \\
V_{s 2}\end{array}$ & $\begin{array}{l}6.05 \\
2.74 \\
2.84\end{array}$ & $\begin{array}{l}6.10 \\
2.87 \\
2.94\end{array}$ & $\begin{array}{l}6.14 \\
2.93 \\
2.98\end{array}$ & $\begin{array}{l}6.17 \\
2.97 \\
3.01\end{array}$ & $\begin{array}{l}6.19 \\
2.99 \\
3.04\end{array}$ & $\begin{array}{l}6.22 \\
3.04 \\
3.07\end{array}$ & $\begin{array}{l}6.24 \\
3.10 \\
3.11\end{array}$ & $\begin{array}{l}6.27 \\
3.10 \\
3.12\end{array}$ & $\begin{array}{l}6.29 \\
3.11 \\
3.12\end{array}$ & $\begin{array}{l}6.32 \\
3.12 \\
3.13\end{array}$ & $\begin{array}{l}\text { Unit 19; Piece 10. Minor disseminated } \\
\text { py. }\end{array}$ \\
\hline $\begin{array}{l}\text { 139-857D-21R-1, } 68-71 \mathrm{~cm} \\
\quad \rho=2.92 \mathrm{~g} / \mathrm{cc} \\
\quad=4.0 \% \\
\text { Medium-grained porphyritic diabase }\end{array}$ & $\mathrm{v}$ & $\begin{array}{l}V_{p} \\
V_{s l} \\
V_{s 2}\end{array}$ & $\begin{array}{l}6.13 \\
3.25 \\
3.24\end{array}$ & $\begin{array}{l}6.25 \\
3.31 \\
3.30\end{array}$ & $\begin{array}{l}6.35 \\
3.36 \\
3.35\end{array}$ & $\begin{array}{l}6.40 \\
3.39 \\
3.38\end{array}$ & $\begin{array}{l}6.44 \\
3.41 \\
3.41\end{array}$ & $\begin{array}{l}6.47 \\
3.44 \\
3.44\end{array}$ & $\begin{array}{l}6.51 \\
3.48 \\
3.48\end{array}$ & $\begin{array}{l}6.54 \\
3.50 \\
3.50\end{array}$ & $\begin{array}{l}6.55 \\
3.51 \\
3.52\end{array}$ & $\begin{array}{l}6.57 \\
3.53 \\
3.54\end{array}$ & $\begin{array}{l}\text { Unit 20A; Piece } 6 \text {. Pheno } 3 \% \text { plag. } \\
\text { GM } 40 \% \text { plag, } 40 \% \text { cpx, tr. oxide. } \\
\text { SM } 10 \% \text { chl, } 2 \% \text { epi, } 3 \% \text { py incl. } \\
\text { large aggregate. }\end{array}$ \\
\hline $\begin{array}{l}\text { 139-857D-21R-1, 73-75 cm } \\
\qquad \begin{array}{l}\rho \\
=2.84 \mathrm{~g} / \mathrm{cc}\end{array} \\
\quad=5.1 \% \\
\text { Medium-grained porphyritic diabase }\end{array}$ & h & $\begin{array}{l}V_{p} \\
V_{s 1} \\
V_{s 2}\end{array}$ & $\begin{array}{l}5.65 \\
3.13 \\
3.08\end{array}$ & $\begin{array}{l}5.73 \\
3.16 \\
3.13\end{array}$ & $\begin{array}{l}5.80 \\
3.21 \\
3.16\end{array}$ & $\begin{array}{l}5.84 \\
3.23 \\
3.19\end{array}$ & $\begin{array}{l}5.87 \\
3.25 \\
3.21\end{array}$ & $\begin{array}{l}5.91 \\
3.27 \\
3.24\end{array}$ & $\begin{array}{l}5.95 \\
3.31 \\
3.27\end{array}$ & $\begin{array}{l}5.95 \\
3.32 \\
3.29\end{array}$ & $\begin{array}{l}5.97 \\
3.33 \\
3.30\end{array}$ & $\begin{array}{l}5.99 \\
3.34 \\
3.31\end{array}$ & $\begin{array}{l}\text { Unit 20A; Piece } 6 .<1 \% \text { disseminated } \\
\text { py; more alteration. }\end{array}$ \\
\hline $\begin{array}{l}139-857 \mathrm{D}-29 \mathrm{R}-2,10-13 \mathrm{~cm} \\
\quad \rho=2.92 \mathrm{~g} / \mathrm{cc} \\
\phi=4.1 \% \\
\text { Medium-grained diabase }\end{array}$ & $\mathrm{v}$ & $\begin{array}{l}V_{p} \\
V_{s t} \\
V_{s 2}\end{array}$ & $\begin{array}{l}5.65 \\
3.16 \\
3.19\end{array}$ & $\begin{array}{l}5.75 \\
3.21 \\
3.24\end{array}$ & $\begin{array}{l}5.81 \\
3.24 \\
3.26\end{array}$ & $\begin{array}{l}5.86 \\
3.27 \\
3.30\end{array}$ & $\begin{array}{l}5.89 \\
3.29 \\
3.32\end{array}$ & $\begin{array}{l}5.92 \\
3.33 \\
3.34\end{array}$ & $\begin{array}{l}5.95 \\
3.36 \\
3.37\end{array}$ & $\begin{array}{l}5.97 \\
3.37 \\
3.38\end{array}$ & $\begin{array}{l}5.99 \\
3.38 \\
3.39\end{array}$ & $\begin{array}{l}6.00 \\
3.39 \\
3.40\end{array}$ & $\begin{array}{l}\text { Unit } 23 \mathrm{~A} \text {; Piece } 2.33 \% \text { plag, } 12 \% \\
\text { cpx, } 1 \% \text { ilm. SM } 35 \% \text { chl, } 10 \% \\
\text { epi, } 9 \% \text { py in large porphyoblast } \\
\text { and } 2 \text { smaller aggregates. }\end{array}$ \\
\hline $\begin{array}{l}139-857 \mathrm{D}-33 \mathrm{R}-1,34-36 \mathrm{~cm} \\
\qquad \begin{array}{l}\rho=2.91 \mathrm{~g} / \mathrm{cc} \\
\phi=5.1 \%\end{array} \\
\text { Fine-grained diabase }\end{array}$ & $\mathrm{v}$ & $\begin{array}{l}V_{p} \\
V_{s l} \\
V_{s 2}\end{array}$ & $\begin{array}{l}6.13 \\
3.06 \\
2.90\end{array}$ & $\begin{array}{l}6.18 \\
3.30 \\
3.11\end{array}$ & $\begin{array}{l}6.22 \\
3.40 \\
3.25\end{array}$ & $\begin{array}{l}6.27 \\
3.43 \\
3.28\end{array}$ & $\begin{array}{l}6.37 \\
3.44 \\
3.33\end{array}$ & $\begin{array}{l}6.39 \\
3.44 \\
3.35\end{array}$ & $\begin{array}{l}6.42 \\
3.45 \\
3.36\end{array}$ & $\begin{array}{l}6.47 \\
3.46 \\
3.37\end{array}$ & $\begin{array}{l}6.49 \\
3.46 \\
3.38\end{array}$ & $\begin{array}{l}6.52 \\
3.47 \\
3.39\end{array}$ & $\begin{array}{l}\text { Unit } 24 \text {; Piece } 6.27 \% \text { plag, } 27 \% \\
\text { cpx, } 2 \% \text { oxide, } 2 \% \text { meso. SM } 25 \% \\
\text { chl, } 10 \% \text { epi, } 2 \% \text { prehnite, } 5 \% \text { py } \\
\text { in dispersed aggregates. }\end{array}$ \\
\hline $\begin{array}{l}139-857 \mathrm{D}-33 \mathrm{R}-1,37-39 \mathrm{~cm} \\
\qquad \begin{array}{l}\rho=2.85 \mathrm{~g} / \mathrm{cc} \\
\phi=6.2 \% \\
\text { Fine-grained diabase }\end{array}\end{array}$ & $\mathrm{h}$ & $\begin{array}{l}V_{p} \\
V_{s l} \\
V_{s 2}\end{array}$ & $\begin{array}{l}5.56 \\
3.07 \\
3.06\end{array}$ & $\begin{array}{l}5.68 \\
3.12 \\
3.13\end{array}$ & $\begin{array}{l}5.75 \\
3.15 \\
3.16\end{array}$ & $\begin{array}{l}5.78 \\
3.18 \\
3.19\end{array}$ & $\begin{array}{l}5.82 \\
3.21 \\
3.22\end{array}$ & $\begin{array}{l}5.85 \\
3.23 \\
3.25\end{array}$ & $\begin{array}{l}5.89 \\
3.27 \\
3.29\end{array}$ & $\begin{array}{l}5.91 \\
3.30 \\
3.30\end{array}$ & $\begin{array}{l}5.92 \\
3.31 \\
3.30\end{array}$ & $\begin{array}{l}5.94 \\
3.32 \\
3.32\end{array}$ & $\begin{array}{l}\text { Unit 24; Piece } 6 . \leq 1 \% \text { oxide, } 7 \% \\
\text { py mostly as elongated blebs in } \\
\text { major vein. }\end{array}$ \\
\hline $\begin{array}{l}139-857 \mathrm{D}-36 \mathrm{R}-1,94-96 \mathrm{~cm} \\
\qquad \rho=2.92 \mathrm{~g} / \mathrm{cc} \\
\quad \phi=4.9 \% \\
\text { Fine-grained diabase }\end{array}$ & $\mathrm{v}$ & $\begin{array}{l}V_{p} \\
V_{s l} \\
V_{s 2}\end{array}$ & $\begin{array}{l}6.14 \\
3.23 \\
3.24\end{array}$ & $\begin{array}{l}6.30 \\
3.31 \\
3.31\end{array}$ & $\begin{array}{l}6.41 \\
3.37 \\
3.37\end{array}$ & $\begin{array}{l}6.47 \\
3.41 \\
3.41\end{array}$ & $\begin{array}{l}6.54 \\
3.44 \\
3.44\end{array}$ & $\begin{array}{l}6.59 \\
3.47 \\
3.48\end{array}$ & $\begin{array}{l}6.64 \\
3.52 \\
3.53\end{array}$ & $\begin{array}{l}6.67 \\
3.54 \\
3.55\end{array}$ & $\begin{array}{l}6.70 \\
3.55 \\
3.56\end{array}$ & $\begin{array}{l}6.72 \\
3.55 \\
3.57\end{array}$ & $\begin{array}{l}\text { Unit } 25 \mathrm{C} \text {; Piece } 14.34 \% \text { plag, } 33 \% \\
\mathrm{cpx}, 1 \% \mathrm{ilm} / \mathrm{mag}, 15 \% \text { meso. } \\
\text { SM } 10 \% \mathrm{chl}, 5 \% \text { act, } 2 \% \text { py. }\end{array}$ \\
\hline $\begin{array}{l}\text { 139-857D-36R-1, 98-101 cm } \\
\qquad \begin{array}{l}\rho=2.77 \mathrm{~g} / \mathrm{cc} \\
\phi=4.4 \%\end{array} \\
\text { Fine-grained diabase }\end{array}$ & $\mathrm{h}$ & $\begin{array}{l}V_{p} \\
V_{s l} \\
V_{s 2}\end{array}$ & $\begin{array}{l}4.82 \\
3.13 \\
3.15\end{array}$ & $\begin{array}{l}5.01 \\
3.14 \\
3.28\end{array}$ & $\begin{array}{l}5.11 \\
3.17 \\
3.43\end{array}$ & $\begin{array}{l}5.18 \\
3.18 \\
3.47\end{array}$ & $\begin{array}{l}5.24 \\
3.19 \\
3.52\end{array}$ & $\begin{array}{l}5.29 \\
3.22 \\
3.54\end{array}$ & $\begin{array}{l}5.35 \\
3.26 \\
3.61\end{array}$ & $\begin{array}{l}5.38 \\
3.28 \\
3.66\end{array}$ & $\begin{array}{l}5.40 \\
3.29 \\
3.68\end{array}$ & $\begin{array}{l}5.43 \\
3.29 \\
3.71\end{array}$ & Unit 25C; Piece $14.1 \%$ py. \\
\hline 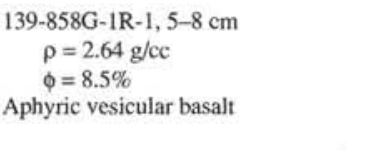 & $\mathrm{v}$ & $\begin{array}{l}V_{p} \\
V_{s l} \\
V_{s 2}\end{array}$ & $\begin{array}{l}5.73 \\
3.04 \\
3.03\end{array}$ & $\begin{array}{l}5.78 \\
3.05 \\
3.05\end{array}$ & $\begin{array}{l}5.81 \\
3.06 \\
3.07\end{array}$ & $\begin{array}{l}5.82 \\
3.07 \\
3.07\end{array}$ & $\begin{array}{l}5.84 \\
3.08 \\
3.08\end{array}$ & $\begin{array}{l}5.86 \\
3.09 \\
3.09\end{array}$ & $\begin{array}{l}5.87 \\
3.10 \\
3.10\end{array}$ & $\begin{array}{l}5.89 \\
3.10 \\
3.10\end{array}$ & $\begin{array}{l}5.90 \\
3.11 \\
3.11\end{array}$ & $\begin{array}{l}5.92 \\
3.11 \\
3.11\end{array}$ & $\begin{array}{l}\text { Unit } 1 \text { C; Piece } 2 \text {. Pheno }<1 \% \text { plag, } \\
<1 \% \text { ol. GM plag microlites and } \\
\text { cryptocryst. matrix. } 3 \% \text { chl- } \\
\text { filled vesicles. SM } 3 \% \text { chl, } 2 \% \text { py. } \\
<1 \% \text { vein filled with chl. }\end{array}$ \\
\hline $\begin{array}{l}139-858 \mathrm{G}-1 \mathrm{R}-1,9-12 \mathrm{~cm} \\
\qquad \begin{array}{l}\rho=2.60 \mathrm{~g} / \mathrm{cc} \\
\quad \phi=8.6 \% \\
\text { Aphyric vesicular basalt }\end{array}\end{array}$ & $\mathrm{h}$ & $\begin{array}{l}V_{p} \\
V_{s l} \\
V_{s 2}\end{array}$ & $\begin{array}{l}6.32 \\
3.06 \\
3.09\end{array}$ & $\begin{array}{l}6.38 \\
3.10 \\
3.11\end{array}$ & $\begin{array}{l}6.40 \\
3.11 \\
3.13\end{array}$ & $\begin{array}{l}6.48 \\
3.13 \\
3.15\end{array}$ & $\begin{array}{l}6.52 \\
3.15 \\
3.17\end{array}$ & $\begin{array}{l}6.55 \\
3.17 \\
3.18\end{array}$ & $\begin{array}{l}* * * \\
3.20 \\
3.21\end{array}$ & $\begin{array}{l}* * * \\
3.21 \\
3.22\end{array}$ & $\begin{array}{l}* * * \\
3.22 \\
3.22\end{array}$ & $\begin{array}{l}* * * \\
3.22 \\
3.23\end{array}$ & $\begin{array}{l}\text { Unit } 1 \text { C; Piece } 2 .<0.2 \% \text { Py. No } \\
\text { apparent veins/cracks. }\end{array}$ \\
\hline
\end{tabular}

Notes: $\mathrm{h}=$ horizontal core oriented perpendicular to core axis; $\mathrm{v}=$ vertical core oriented parallel to core axis. $V_{p}=$ compressional-wave velocity; $V_{s l}=$ shear wave propagating perpendicular to minicore alignment direction; $V_{s 2}=$ shear wave propagating parallel to minicore alignment direction. Velocities with asterisks extrapolated from curve fits. Pheno = phenocrysts; $\mathrm{GM}=\mathrm{groundmass} ; \mathrm{SM}=$ secondary

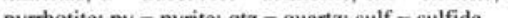

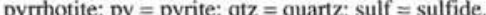


Table 2. Compressional- and shear-wave velocities for samples from Holes $857 \mathrm{C}$ and $857 \mathrm{D}$, and compositional descriptions, measured to $600 \mathrm{MPa}$ at the Purdue Rock Physics Laboratory.

\begin{tabular}{|c|c|c|c|c|c|c|c|c|c|c|c|c|c|}
\hline \multirow[b]{2}{*}{ Sample Description } & \multirow[b]{2}{*}{ Direction } & \multirow{2}{*}{$\begin{array}{l}\text { Velocity } \\
(\mathrm{km} / \mathrm{s})\end{array}$} & \multicolumn{10}{|c|}{ Pressure (MPa) } & \multirow[b]{2}{*}{ Comments } \\
\hline & & & 20 & 40 & 60 & 80 & 100 & 200 & 300 & 400 & 500 & 600 & \\
\hline $\begin{array}{l}139-857 \mathrm{C}-59 \mathrm{R}-3,56-58 \mathrm{~cm} \\
\quad \rho=2.86 \mathrm{~g} / \mathrm{cc} \\
\text { Coarse-grained diabase }\end{array}$ & h & $V_{p}$ & $\begin{array}{l}4.75 \\
2.77\end{array}$ & $\begin{array}{l}5.00 \\
2.91\end{array}$ & $\begin{array}{l}5.18 \\
3.01\end{array}$ & $\begin{array}{l}5.31 \\
3.08\end{array}$ & $\begin{array}{l}5.42 \\
3.13\end{array}$ & $\begin{array}{l}5.74 \\
3.27\end{array}$ & $\begin{array}{l}5.88 \\
3.33\end{array}$ & $\begin{array}{l}5.96 \\
3.36\end{array}$ & $\begin{array}{l}3.01 \\
3.39\end{array}$ & $\begin{array}{l}6.05 \\
3.41\end{array}$ & $\begin{array}{l}\text { Unit } 1 \text {; Piece } 2.40 \% \text { plag, } 38 \% \text { cpx, } \\
2 \% \text { ilm/mag. SM } 1 \% \text { clay, } 12 \% \text { chl, } \\
4 \% \text { epi, } 1 \% \text { act, } 1 \% \text { prehnite, } 1 \% \\
\text { po/cpo. }\end{array}$ \\
\hline $\begin{array}{l}139-857 \mathrm{C}-68 \mathrm{R}-2,51-53 \mathrm{~cm} \\
\quad \rho=2.68 \mathrm{~g} / \mathrm{cc} \\
\text { Medium-grained diabase }\end{array}$ & h & $V_{p}$ & $\begin{array}{l}3.59 \\
2.01\end{array}$ & $\begin{array}{l}3.91 \\
2.22\end{array}$ & $\begin{array}{l}4.13 \\
2.37\end{array}$ & $\begin{array}{l}4.61 \\
2.48\end{array}$ & $\begin{array}{l}4.45 \\
2.58\end{array}$ & $\begin{array}{l}4.92 \\
2.85\end{array}$ & $\begin{array}{l}5.16 \\
2.98\end{array}$ & $\begin{array}{l}5.32 \\
3.05\end{array}$ & $\begin{array}{l}5.42 \\
3.10\end{array}$ & $\begin{array}{l}5.49 \\
3.14\end{array}$ & $\begin{array}{l}\text { Unit } 12 \text {; Piece } 6.36 \% \text { plag, } 39 \% \mathrm{cpx} \text {, } \\
3 \% \text { ilm. SM } 15 \% \mathrm{chl}, 5 \% \text { epi, } 1 \% \text { act, } \\
1 \% \text { py. }\end{array}$ \\
\hline $\begin{array}{l}\text { 139-857D-3R-2, } 64-66 \mathrm{~cm} \\
\qquad \rho=2.81 \mathrm{~g} / \mathrm{cc} \\
\text { Medium-grained phyric diabase }\end{array}$ & $\mathrm{h}$ & $\begin{array}{l}V_{p} \\
V_{s}\end{array}$ & $\begin{array}{l}4.18 \\
2.44\end{array}$ & $\begin{array}{l}4.51 \\
2.59\end{array}$ & $\begin{array}{l}4.74 \\
2.70\end{array}$ & $\begin{array}{l}4.92 \\
2.78\end{array}$ & $\begin{array}{l}5.07 \\
2.85\end{array}$ & $\begin{array}{l}5.48 \\
3.05\end{array}$ & $\begin{array}{l}5.66 \\
3.15\end{array}$ & $\begin{array}{l}5.76 \\
3.21\end{array}$ & $\begin{array}{l}5.83 \\
3.25\end{array}$ & $\begin{array}{l}5.87 \\
3.28\end{array}$ & $\begin{array}{l}\text { Unit I4B; Piece } 8 \text {. Pheno } 3 \% \text { plag. } \\
\text { GM } 41 \% \text { plag, } 42 \% \mathrm{cpx}, 3 \% \text { ilm/mag. } \\
\text { SM } 6 \% \text { chl, } 2 \% \text { epi, } 2 \% \text { act, } 1 \% \text { sulf, } \\
\text { tr. qtz. }\end{array}$ \\
\hline $\begin{array}{l}\text { 139-857D-18R-1, } 49-51 \mathrm{~cm} \\
\qquad \rho=2.84 \mathrm{~g} / \mathrm{cc} \\
\text { Medium-grained porphyritic diabase }\end{array}$ & h & $V_{p}$ & $\begin{array}{l}5.07 \\
2.95\end{array}$ & $\begin{array}{l}5.33 \\
3.04\end{array}$ & $\begin{array}{l}5.52 \\
3.10\end{array}$ & $\begin{array}{l}5.63 \\
3.14\end{array}$ & $\begin{array}{l}5.70 \\
3.18\end{array}$ & $\begin{array}{l}5.88 \\
3.29\end{array}$ & $\begin{array}{l}5.94 \\
3.35\end{array}$ & $\begin{array}{l}5.99 \\
3.38\end{array}$ & $\begin{array}{l}6.02 \\
3.41\end{array}$ & $\begin{array}{l}6.05 \\
3.43\end{array}$ & $\begin{array}{l}\text { Unit } 19 \text {; Piece } 4 \text { A. } 45 \% \text { plag, } 34 \% \text { cpx, } \\
3 \% \text { py. SM } 6 \% \text { chl, } 5 \% \text { epi, } 3 \% \text { py, } \\
3 \% \text { albite, } 1 \% \text { sphene, tr. qtz. } 5 \% \text { vugs } \\
\text { with epi, chl, py, cpy. }\end{array}$ \\
\hline $\begin{array}{l}\text { 139-857D-24R-2, } 38-40 \mathrm{~cm} \\
\qquad \rho=2.86 \mathrm{~g} / \mathrm{cc} \\
\text { Medium-grained phyric diabase }\end{array}$ & h & $V_{p}$ & $\begin{array}{l}4.97 \\
2.91\end{array}$ & $\begin{array}{l}5.25 \\
3.05\end{array}$ & $\begin{array}{l}5.42 \\
3.14\end{array}$ & $\begin{array}{l}5.54 \\
3.20\end{array}$ & $\begin{array}{l}5.63 \\
3.24\end{array}$ & $\begin{array}{l}5.85 \\
3.33\end{array}$ & $\begin{array}{l}5.95 \\
3.37\end{array}$ & $\begin{array}{l}6.01 \\
3.39\end{array}$ & $\begin{array}{l}6.06 \\
3.41\end{array}$ & $\begin{array}{l}6.10 \\
3.43\end{array}$ & $\begin{array}{l}\text { Unit } 21 \text {; Piece } 5 \text {. Pheno } 1 \% \text { plag. GM } \\
44 \% \text { plag, } 32 \% \text { cpx, } 3 \% \text { ilm/mag. SM } \\
15 \% \text { chl, } 5 \% \text { epi. } 3-5 \% \text { chl-filled } \\
\text { vesicles nearby. }\end{array}$ \\
\hline $\begin{array}{l}\text { 139-857D-35R-1, } 102-104 \mathrm{~cm} \\
\quad p=2.92 \mathrm{~g} / \mathrm{cc} \\
\text { Medium-grained metadiabase }\end{array}$ & h & $V_{p}$ & $\begin{array}{l}5.74 \\
3.11\end{array}$ & $\begin{array}{l}5.67 \\
3.19\end{array}$ & $\begin{array}{l}5.81 \\
3.24\end{array}$ & $\begin{array}{l}5.90 \\
3.28\end{array}$ & $\begin{array}{l}5.97 \\
3.31\end{array}$ & $\begin{array}{l}6.11 \\
3.39\end{array}$ & $\begin{array}{l}6.18 \\
3.43\end{array}$ & $\begin{array}{l}6.24 \\
3.46\end{array}$ & $\begin{array}{l}6.28 \\
3.48\end{array}$ & $\begin{array}{l}6.31 \\
3.49\end{array}$ & $\begin{array}{l}\text { Unit } 25 \mathrm{~B} \text { : Piece } 14 \mathrm{~A} .35 \% \mathrm{cpx}, 25 \% \\
\text { plag, } 4 \% \text { ilm/mag. SM } 25 \% \mathrm{chl} \text {, } \\
5 \% \text { act, } 1 \% \text { epi, } 5 \% \text { py. }\end{array}$ \\
\hline $\begin{array}{l}139-857 \mathrm{D}-35 \mathrm{R}-1,106-109 \mathrm{~cm} \\
\quad \rho=2.91 \mathrm{~g} / \mathrm{cc} \\
\text { Medium-grained metadiabase }\end{array}$ & $\mathrm{v}$ & $V_{p}$ & $\begin{array}{l}5.40 \\
3.08\end{array}$ & $\begin{array}{l}5.66 \\
3.19\end{array}$ & $\begin{array}{l}5.81 \\
3.26\end{array}$ & $\begin{array}{l}5.91 \\
2.99\end{array}$ & $\begin{array}{l}5.97 \\
3.34\end{array}$ & $\begin{array}{l}6.12 \\
3.42\end{array}$ & $\begin{array}{l}6.18 \\
3.45\end{array}$ & $\begin{array}{l}6.23 \\
3.48\end{array}$ & $\begin{array}{l}6.26 \\
3.50\end{array}$ & $\begin{array}{l}6.29 \\
6.51\end{array}$ & $\begin{array}{l}\text { Unit } 25 \mathrm{~B} \text {; Piece } 14 \mathrm{~A} \text {. As above, with } \\
\text { py along fracture planes. }\end{array}$ \\
\hline
\end{tabular}

Notes: $\mathrm{h}=$ horizontal core oriented perpendicular to core axis; $\mathrm{v}=$ vertical core oriented parallel to core axis. $V_{p}=$ compressional-wave velocity; $V_{s}=$ shear-wave velocity. Pheno $=$ phenocrysts; $\mathrm{GM}=$ groundmass; $\mathrm{SM}=$ secondary minerals; $\mathrm{tr}=$ trace. Act = actinolite; $\mathrm{chl}=$ chlorite $; \mathrm{cpx}=$ clinopyroxene; $\mathrm{cpy}=$ chalcopyrite; epi $=$ epidote; ilm = ilmenite; mag = magnetite; plag = plagioclase; $\mathrm{py}=$ pyrite; qtz = quartz; sulf = sulfide. Comments from shipboard visual core descriptions and nearby thin-section descriptions.

from mid-ocean ridge basalts (MORB). The most interesting result of the study is that the physical properties of these sill rocks, exposed to variable temperatures and probable focused hydrothermal fluid flow, are no different from relatively fresh, unweathered MORB.

\section{ANALYTICAL METHODS AND SAMPLE PREPARATION}

Horizontally and vertically oriented igneous rock minicores were drilled perpendicular and parallel, respectively, to the axis of the core. The 2.54-cm-diameter samples were stored in seawater and imperfections in vertical samples were patched with two-part epoxy. Lengths of the right-circular cylinders were measured with a digital caliper micrometer. All samples were saturated under vacuum in a bell jar for 24 to $48 \mathrm{hr}$ prior to measurement. The ends were trimmed flat and parallel to within $0.008 \mathrm{~cm}$. Polished thin sections for petrographic analyses were made from 21 sample stubs.

The high-pressure velocity system and experimental methodology used for seven measurements carried out at Purdue University at confining pressure from 20 to $600 \mathrm{MPa}$ are described in detail by Christensen (1985). A lower-pressure velocimeter of similar geometry was used for measurements made at the Rosenstiel School of Marine and Atmospheric Science (RSMAS). Velocities of 19 diabase and two basalt samples were measured at confining pressures from 3 to 100 $\mathrm{MPa}$ and a constant pore pressure of $2 \mathrm{MPa}$ (Fig. 1). A unique feature of this $1-\mathrm{MHz}$ transducer system is that two orthogonal shear waves (S1 and S2) are propagated through the sample along with a single compressional wave. The minicores were aligned so that the $\mathrm{S} 2$ wave propagated in the plane defined by the orientation marks whereas the $\mathrm{S} 1$ wave propagated in a plane oriented $90^{\circ}$ from the S2 plane. The precision of $V_{p}$ and $V_{s}$ measurements for intact rock samples is approximately $1 \%$ for samples measured at Purdue and approximately $1 \%$ to $2 \%$ at RSMAS.

Bulk densities for the seven minicores sent to Purdue were calculated from their dimensions and mass. Porosity data for these samples are not available. RSMAS samples were sent to the shorebased physical properties laboratory at the Ocean Drilling Program. The masses of saturated and dry minicores were determined with an electronic balance $( \pm 0.010 \mathrm{~g}$ accuracy), and dry volumes were obtained with the same model Quantachrome helium Penta-Pycnometer utilized for shipboard measurements ( $\pm 0.03 \%$ accuracy). Resultant densities are good to $0.2 \%\left( \pm 0.006 \mathrm{~g} / \mathrm{cm}^{3}\right)$. Salinity-corrected wet-bulk densities and porosities were recalculated from shipboard data and standard ODP formulae that utilize dry volumes in order to be consistent with the shorebased dataset (Davis, Mottl, Fisher et al., 1992).

Petrographic analyses for a subset of samples were made with a Leica Quantimet 500 Image Analysis computer system interfaced with a stereoscope and a reflected/transmitted light microscope. Point counts provided quantitative estimates of mineral constituents that have velocities and densities different than those of plagioclase and clinopyroxene, which are the dominant primary minerals (Table 3 ). The percentage, orientation, and average width of filled veins and microcracks per thin section were determined with the stereoscope. Reflected light microscopy was used to discriminate between and determine percentages of oxide and sulfide minerals; nonreflective mineral percentages were also measured. The patchy, intergrown dis- 


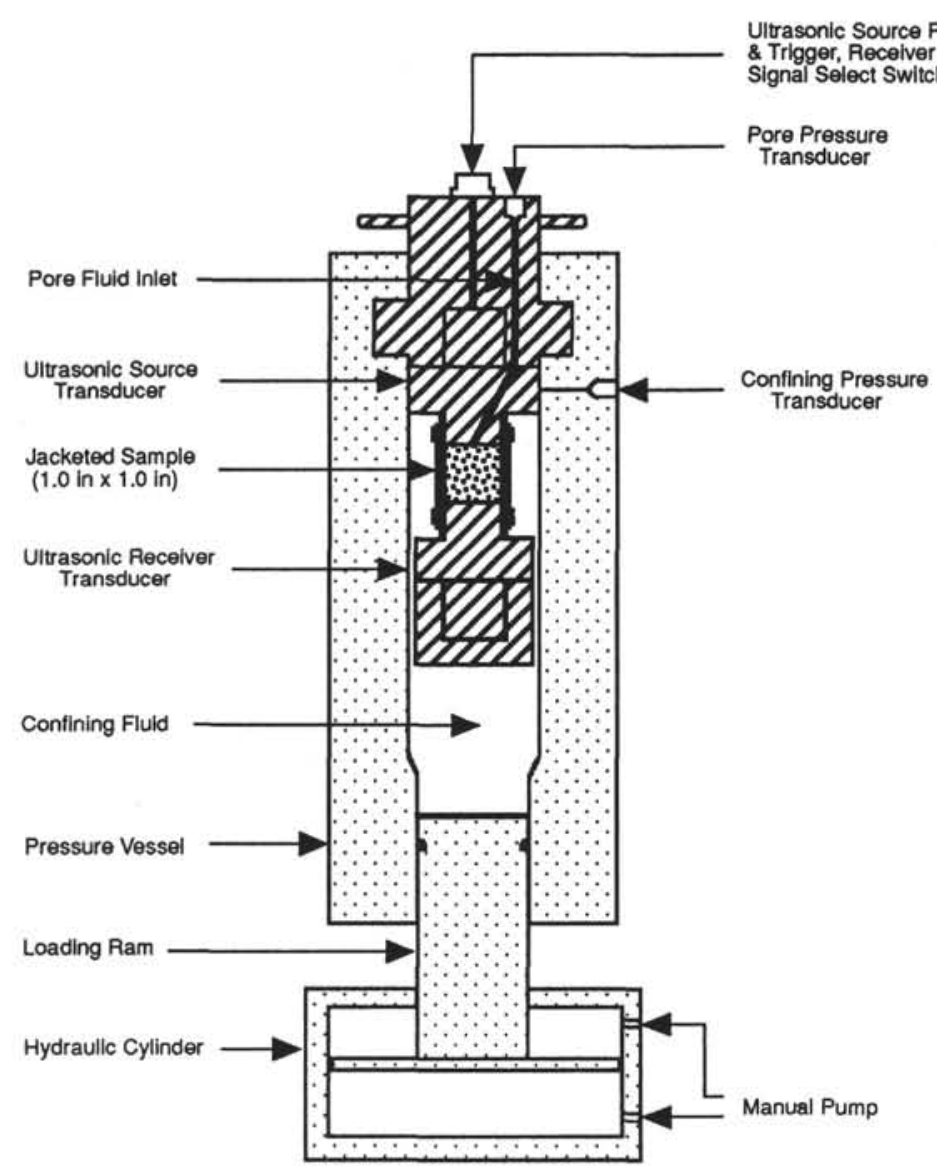

tribution of many alteration and secondary products precluded identification of constituent minerals and resulted in the incorporation of minor amounts of spherulitic, dark-colored, nonsecondary minerals as part of these counts. These are considered the most subjective of all the measurements. The percentage of sphene (titanite), which is useful in the back-calculation of the original amount of oxides present, was also determined. The resolution of this method is based on the pixel size of the video image of the sample. The smallest mineral components analyzed in this study are the oxides; the detection limit is $0.001 \%$ based on our analysis and another made with ODP Hole 735B gabbros (J. Natland, pers. comm., 1993).

\section{SAMPLE DESCRIPTIONS}

A majority of the samples were from cores recovered from a series of mafic sills interbedded with altered and indurated sediment drilled in the lower $96.6 \mathrm{~m}$ of Hole $857 \mathrm{C}$ (471.1-567.7 m below seafloor [mbsf]) and between 581.1 and 936.2 mbsf in reentry Hole $857 \mathrm{D}$ (Fig. 2). Thicknesses of the sills ranged from 1 to $25 \mathrm{~m}$; core recovery and resistivity, spontaneous potential, natural gamma-ray and Formation MicroScanner (FMS) logs (Shipboard Scientific Party, 1992b, figs. 53,87 and 88) suggested penetration of 12 sills in Hole 857C and 30 sills in Hole 857D. Sampling locations were selected with the help of shipboard petrologists to ensure that rocks representative of the cored sills were obtained. Rock compositions were determined from shipboard visual core descriptions and shipboard and shorebased thinsection analyses.

Highly metamorphosed chilled margins near sediment contacts and a progressive increase in grain size away from these margins to very coarse-grained interiors in major sills are common at Site 857 (Shipboard Scientific Party, 1992b). The fine-grained rock is intensely veined (Sample 139-857D-1R-1) and vesicular, and plagioclase phenocrysts are dominant. Heterogeneous textures and medium to coarse
Figure 1. Schematic diagram of the sample assembly and pressure vessel for the RSMAS 100-MPa velocimeter.

grain sizes characterize the 15 minicores sampled from relatively fresh sill interiors. In these rocks, plagioclase is poikilitically intergrown with clinopyroxene and lesser ilmenite, magnetite, and mesostasis. Upper-zeolite to greenschist facies alteration is observed throughout the sills and occurs as recrystallization of plagioclase and mesostasis to epidote, chlorite, albite, and actinolite; wall-rock alteration associated with infilling of veins with chlorite, epidote, quartz, zeolites, and sulfide minerals; and as sulfide (especially disseminated pyrite) deposition. Veins and microfractures are abundant in the entire Hole 857C section and in the upper $143 \mathrm{~m}$ of Hole 857D (581.5$724.5 \mathrm{mbsf}$ ).

The two samples from Section 139-856A-14X-1 were described as massive, fine- to medium-grained, non- to slightly altered diabase from the upper portion of a sill that is notable for the variability observed in the grain size and crystallinity of the groundmass (Shipboard Scientific Party, 1992a). The core piece from which the two minicores from Section 139-858G-1R-1 were taken was classified as lithologic Subunit IC (Shipboard Scientific Party, 1992c) and described as variolitic, fine-grained basalt with dominant plagioclase phenocrysts and common chlorite-filled vesicles. Site 858 igneous rocks are believed to have formed as numerous, extrusive massive flows, and are similar in composition to Site 857 sill rocks.

\section{FACTORS AFFECTING SEISMIC PROPERTIES OF LEG 139 MAFIC ROCKS}

Significant differences in physical and compositional properties are apparent for most of the oriented sample pairs separated by only 1 to $3 \mathrm{~cm}$ in the split-core section, despite attempts to obtain texturally similar samples. Core pieces with obvious surface inhomogeneities were not selected unless the features seemed characteristic of in-situ structure; the scale of drilling-induced fractures permitted discrimination against core pieces with apparent disturbance features at the 


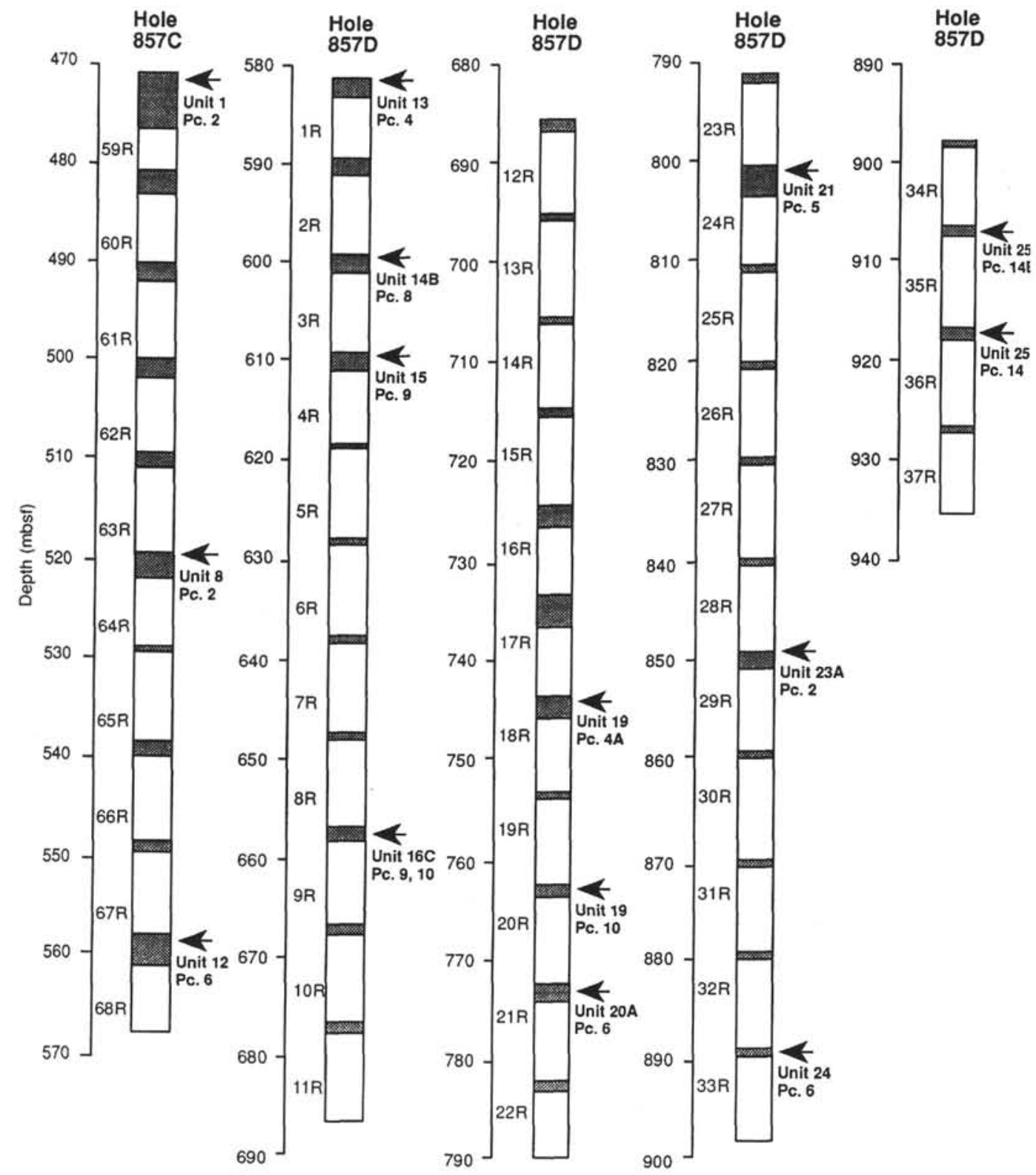

Figure 2. Location and thicknesses of igneous units penetrated in mafic sill/intercalated sediment complex in Holes 857C and 857D (after fig. 51, Shipboard Scientific Party, 1992b). Sample locations are approximated by arrows to the right of columns.

sampling table. Filled veins and partially filled cracks noted along the long axes of Site 857 diabase minicores were considered inherent to the rock, and rapid grain-size changes and alteration mineral distributions were observed in thin sections. Petrographic image analyses provided modal percentages of mineral constituents for only a single two-dimensional circular slice per sample, and variability in the minicores' third dimension is unknown except for surface characteristics. This lack of information limits the interpretation of velocity variations since the acoustic signal is transmitted through the long axis of the rock cylinder. However, inferences about preferred sound wave travel paths through the sample can be made based on the frequency and orientation of observed cracks and veins.

\section{Porosity}

Porosities calculated for Site 857 diabases range from $3.96 \%$ to $14.38 \%$ (Table 1) with a mean for 17 samples of $7.26 \%$. All are well above the average of $2 \%$ reported for extrusive basalts of upper Layer 2 at Hole 504B (Christensen et al., 1989). However, our mean value is similar to the mean porosity of $7.8 \%( \pm 4.1 \%)$ computed by Hyndman and Drury (1976) for 85 young, relatively unweathered vesicular basalts recovered during Deep Sea Drilling Program (DSDP) Leg 37 at $37^{\circ} \mathrm{N}$ on the Mid-Atlantic Ridge (MAR). A plot of shipboard and shore-based Site 857 porosities and wet-bulk densities as functions of depth (Fig. 3) shows the porosity variability with depth in both sills and turbidite sediment interbeds. Data from Hole 857D are appended to the Hole $857 \mathrm{C}$ profile to present a continuous section through the sill complex. Several trends are evident despite the low sampling density resulting from poor core recovery, averaging $15 \%$, and preferential recovery of sill rocks over sediment. Decreasing sediment porosities from 700.0 to 740.0 mbsf may be related to the intrusion of a major sill at the base of this interval. Wet-bulk densities vary inversely to porosities in the previous examples in response to the replacement of primary minerals by alteration and secondary minerals. High shipboard porosities and low densities below $500.0 \mathrm{mbsf}$ from Sections 139-857C-62R-1, -64R-1, -64R-2, -68R-2 and -68R-3 can be correlated with numerous sulfide-filled fractures that are concentrated in highly altered sill margins and extend into the fresher 
Table 3. Modal percentages of oxide, sulfide, and non-reflective (secondary) minerals, and percentage of veins and/or cracks for thin sections from Hole 857D and 858G samples.

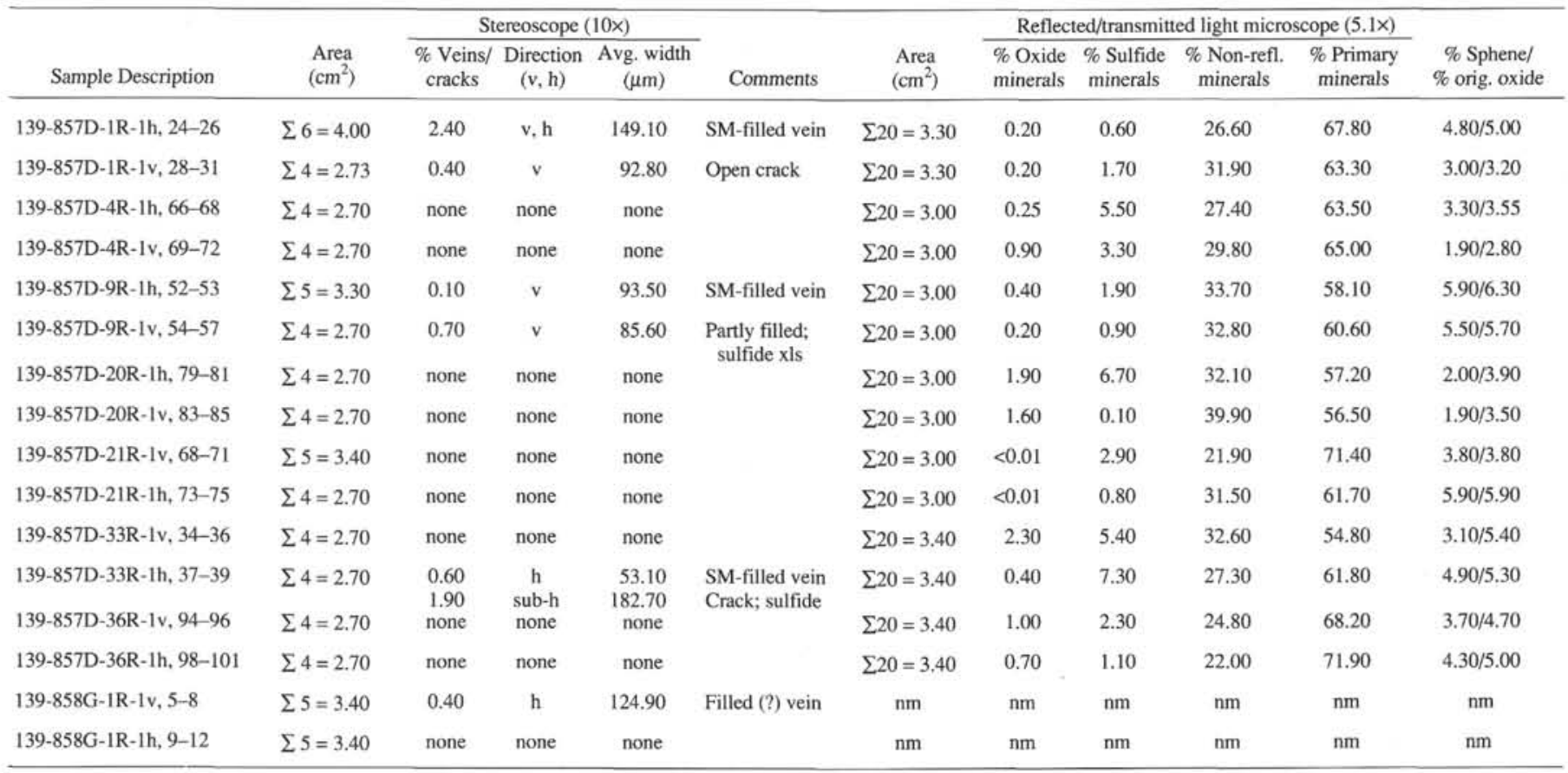

Notes: $x$ ls = crystals; non-reflective/secondary minerals $(\mathrm{SM})=$ chlorite, talc, actinolite, epidote, clay; oxide minerals = ilmenite, magnetite; sulfide minerals = pyrite, pyrrhotite, chalcopyrite primary minerals = plagioclase, $\mathrm{cpx}$, olivine, mesostasis; $\mathrm{h}=$ horizontal vein/crack oriented perpendicular to core axis; $\mathrm{v}=\mathrm{vertical}$ vein $/ \mathrm{crack}$ oriented parallel to core axis; $\mathrm{nm}=\mathrm{not}$ measured.

rock (Shipboard Scientific Party, 1992b). Abundant vesicles, filled most commonly with chlorite, are typically coincident in these zones. A subtle decrease in porosities and increase in densities with depth for both igneous rocks and sediments is evident.

Examples of different porosity types seen in Site 857 diabase thin sections are shown in Plates 1 and 2. The photomicrographs of Plate 1 were taken from a fine-grained leucocratic diabase from the chilled margin of the first sill penetrated beneath the sediment column at Hole $857 \mathrm{D}$, and the most porous samples in this study. A network of randomly oriented open and filled cracks dominates the horizontal sample (Pl. 1, Figs. 1 and 2; Sample 139-857D-1R-1, 24-26 cm) and is less abundant in the vertical sample (PI. 1, Figs. 3 and 4; Sample 139-857D-1R-1, 28-31 cm) farther away from the margin. The most common type of discontinuity in both samples is filled veins that travel through fractured plagioclase crystals and the altered groundmass (Pl. 1, Fig. 1). Void spaces are associated with the plagioclase fragments, and the veins are filled with a fine-grained, fibrous mineral resembling chlorite (PI. 1, Fig. 2). Some cracks are partially to fully filled with secondary plagioclase (albite?) crystals or a combination of larger sulfide and minor plagioclase grains (Pl. 1, Figs. 3 and 4). Microfractures in the coarser-grained samples obtained from sill interiors are fewer in number and typically partly filled with aggregates of macroscopic sulfide crystals (Pl. 2, Fig. 1; Sample 139-857D-9R$1,54-57 \mathrm{~cm})$. The well-formed sulfide crystal packages are interconnected by either narrow filled veins (Pl. 2, Fig. 1) or open cracks (PI. 2, Fig. 3; Sample 139-857D-33R-1, 37-39 cm).

$V_{p}$ data for the Site 857 samples at $100 \mathrm{MPa}$ (solid line) show a decrease in velocities with increasing crack porosity (Fig. 4) that is similar to the trend for shipboard samples with velocities measured at atmospheric pressures (dashed line). The application of increasing confining pressure during measurement reduces the aperture of drilling-related cracks and some endemic cracks; thus both compressional-and shear-wave velocities increase as porosity effects decrease. Some cracks close as increasing pressure is applied and only partially reopen after pressures are reduced (Birch, 1961), resulting in velocity hysteresis, as seen between 10 and $50 \mathrm{MPa}$ in Sample 139-857D-9R$1,51-53 \mathrm{~cm}$ (Fig. 5). Thermal contraction cracks, vesicles, and intergranular pores may not close as pressure is increased, and the presence of alteration products may also inhibit closure (Christensen et al., 1979; Christensen and Wilkens, 1982). Site 857 sill porosities are unu- sually high and variable owing to the presence of unsealed cracks and void spaces of various aspect ratios (Wilkens et al., 1991) and differing percentages of alteration products.

\section{Density}

The mean bulk density for all Leg 139 samples, shown in the $V_{p}$-density plot of Figure 6 , is $2.81 \mathrm{~g} / \mathrm{cm}^{3}$. The mean $V_{p}$ is $5.77 \mathrm{~km} / \mathrm{s}$ and the mean $V_{s}$ is $3.16 \mathrm{~km} / \mathrm{s}$ at $100 \mathrm{MPa}$, the highest pressure at which all samples were measured. We compared the values for our hydrothermal basement samples to two large datasets obtained from mid-ocean ridge basement rocks. $V_{p}$-density and $V_{s}$-density systematics at $50 \mathrm{MPa}$ for Leg 139 samples and 77 DSDP Atlantic and Pacific Layer 2 basalts of varied ages and alteration are shown in Figure 7, adapted from Christensen and Salisbury (1975, fig. 16). The linear (correlation coefficient $=0.96$ ) and nonlinear regression solutions apply to their data only. Our samples have a mean $V_{p}$ of $5.65 \mathrm{~km} / \mathrm{s}$ and a mean $V_{s}$ of $3.07 \mathrm{~km} / \mathrm{s}$ at $50 \mathrm{MPa}$. For given densities, our data exhibit a wider range of velocities than shown by the DSDP basalts. A fresh tholeiitic basalt sample dredged from the western edge of the Juan de Fuca Ridge median valley (Christensen, 1970), shown by the solid triangle in Figure 7, also falls off the DSDP $V_{p}$-density trend. The Leg 139 mean bulk density of $2.81 \mathrm{~g} / \mathrm{cm}^{3}$ is the same as the mean bulk density of $2.795 \mathrm{~g} / \mathrm{cm}^{3}\left( \pm 0.045 \mathrm{~g} / \mathrm{cm}^{3}\right)$ calculated by Hyndman and Drury (1976) for 85 fresh, unweathered, mostly vesicular MAR basalts. Our mean $V_{p}$ of $5.65 \mathrm{~km} / \mathrm{s}$ falls within the lower range of the MAR values at $50 \mathrm{MPa}$ of $5.94 \mathrm{~km} / \mathrm{s}( \pm 0.34 \mathrm{~km} / \mathrm{s})$. Mean $V_{s}$ values at $50 \mathrm{MPa}$ are $3.06 \mathrm{~km} / \mathrm{s}$ for Leg 139 samples and $3.27 \mathrm{~km} / \mathrm{s}( \pm 0.15$ $\mathrm{km} / \mathrm{s}$ ) for the MAR rocks. 


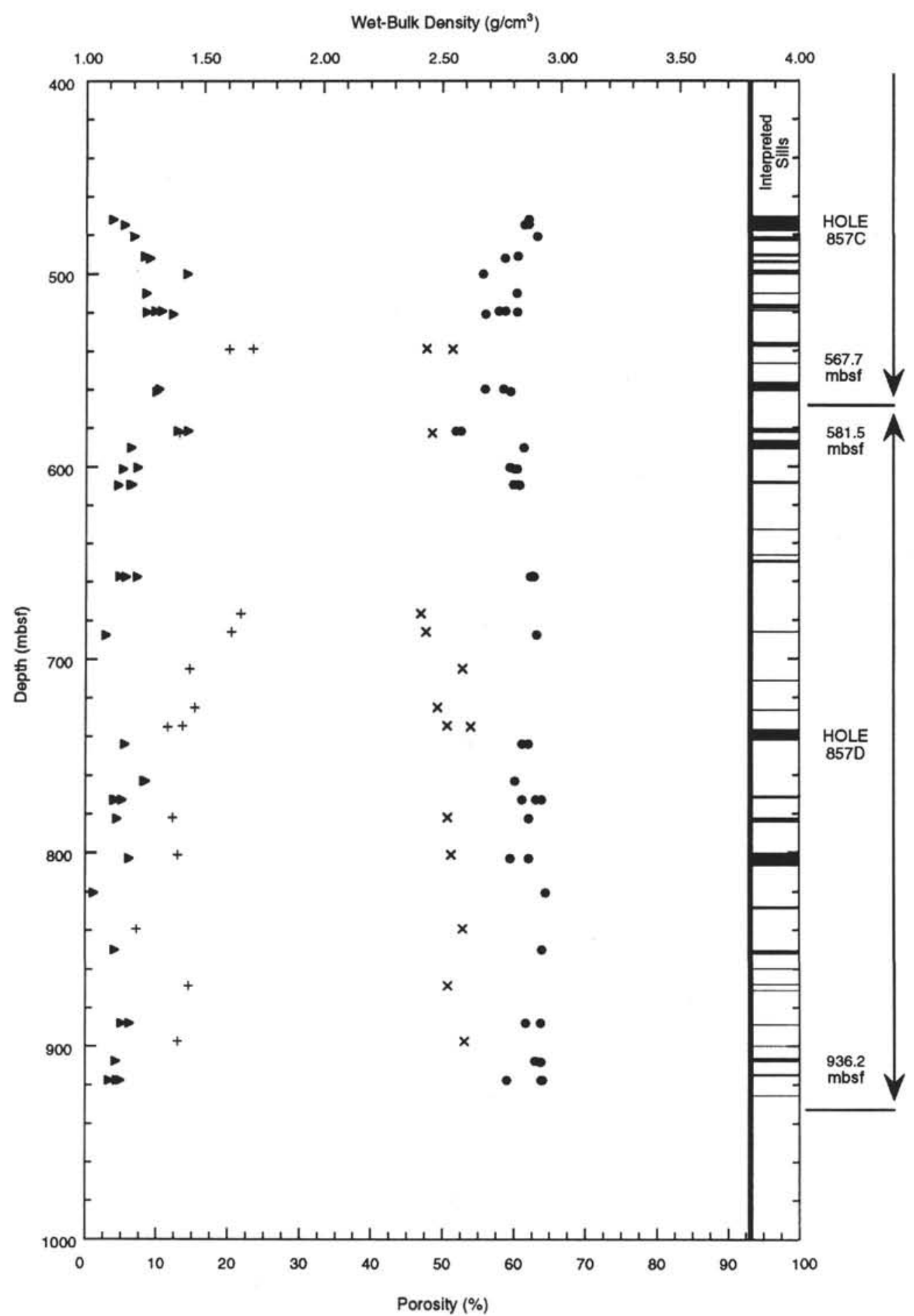

Figure 3. Wet-bulk densities (WBD) and porosities vs. depth for a Site 857 composite sill complex section. Approximate locations and representative thicknesses of interpreted sills are at the right of the figure. Sill WBD are represented by circles and sediment WBD by x's. Triangles are sill porosities and pluses indicate sediment porosities.

Of the 27 Leg 139 samples, 21 from Holes $857 \mathrm{C}$ and $857 \mathrm{D}$ plot within or near the range of relatively young, unweathered basalts that make up the higher $V_{p}$-higher density portions of the Christensen and Salisbury (1975) relations (Fig. 7). None fall near their low $V_{p}$-low density data, which were measured on older basalts affected by lowtemperature weathering. Similarly, the general agreement of the mean $V_{p}$ for our samples, which have been modified by low- to hightemperature alteration processes, with the average $V_{p}$ range of fresh, unweathered MAR rocks is intriguing. These results suggest that different emplacement and alteration histories can produce mineral assemblages with similar physical properties.

Birch (1961) showed empirically that the mean atomic weights of common rocks vary only slightly from 21 and that velocity can be assumed to linearly correlate with density for a given mean atomic weight. This law provides a convenient, first-order method of examining velocity-density relationships in the absence of chemical com- 


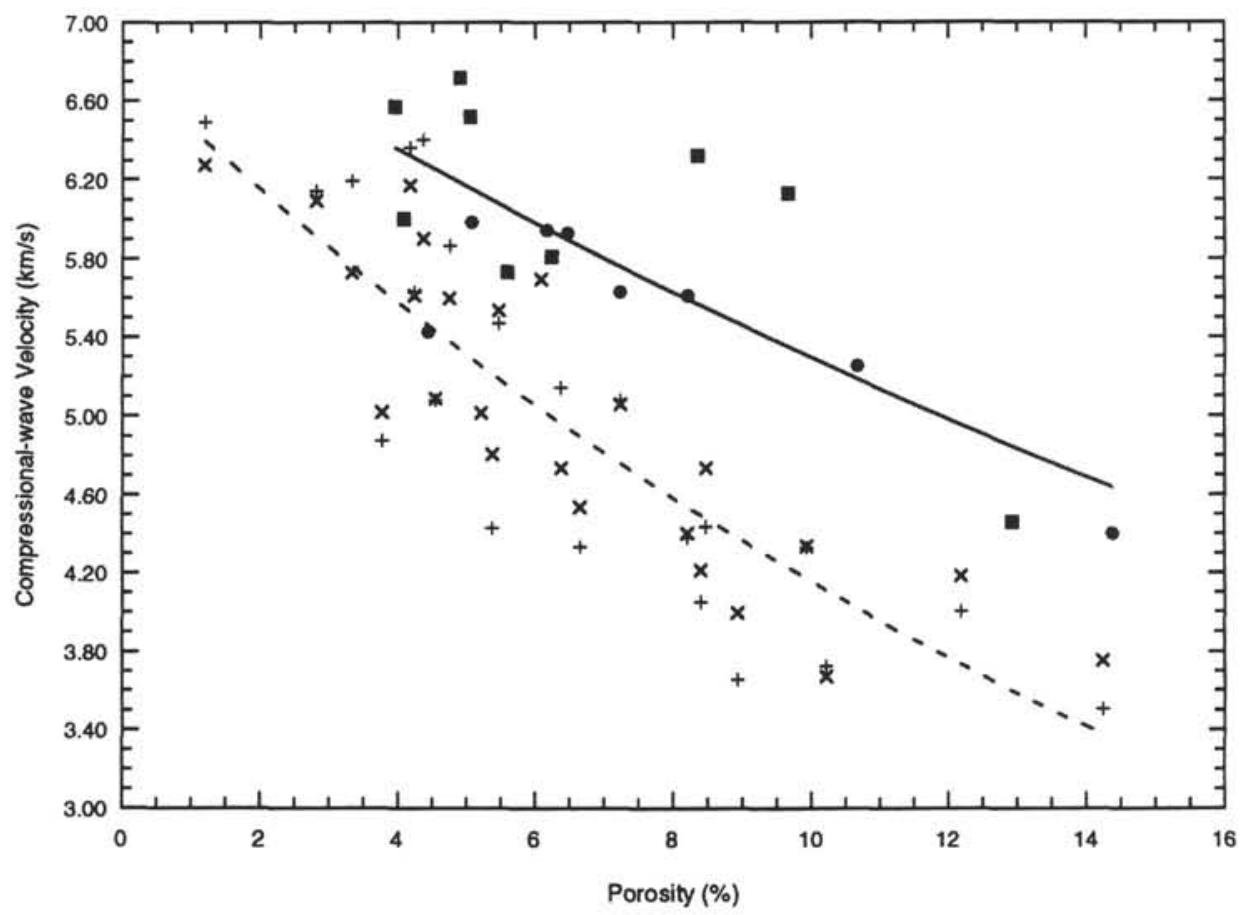

Figure 4. Effect of crack porosity on compressional-wave velocities measured for Site 857 samples at $100 \mathrm{MPa}$ (circles, squares, and solid best-fit line) and atmospheric pressure (x's, pluses, and dashed best-fit line). Circles and x's represent horizontal minicores; squares and pluses represent vertical minicores.

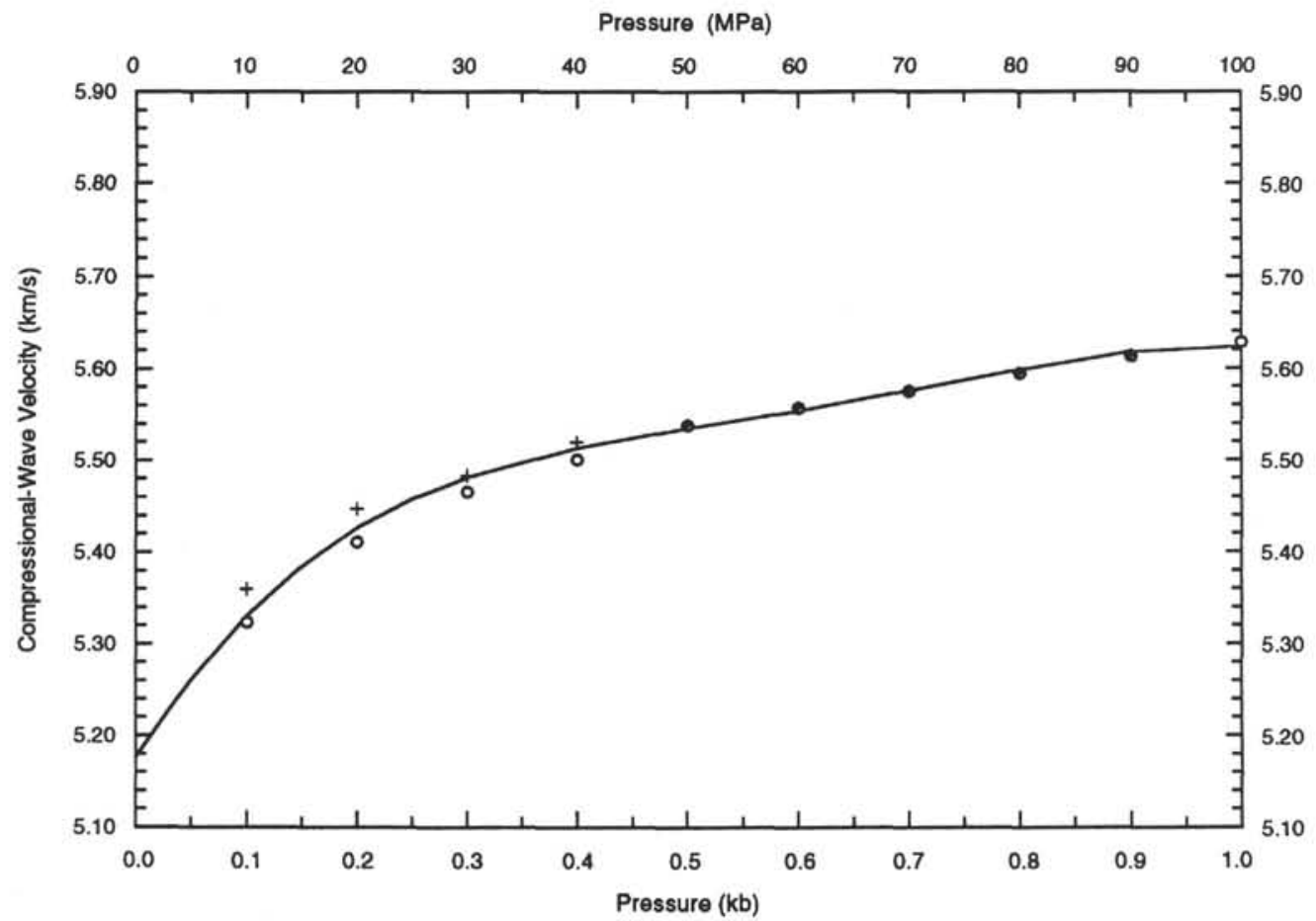

Figure 5. Compressional-wave velocity as a function of confining pressure. Hysteresis between $V_{p}$ measured as pressure increases (circles) and as pressure decreases (pluses) is the result of crack porosity response.

position data (Christensen and Salisbury, 1975), as is the case here, and can be expressed graphically as a $V_{p}$-density plot with lines of constant mean atomic weight $(\mathrm{m})$. The mean atomic weights of ironfree silicates fall near 20 . Increasing iron and, to a lesser extent, calcium contents in constituent minerals shift values to the $\mathrm{m}=21$ region, and increasing iron and titanium contents cause deviation toward the $m=22$ region (Birch, 1961, table 13). Birch's law is based on unsaturated aggregate velocities measured at high confining pres- sures of $1000 \mathrm{MPa}$; its usefulness for rock data obtained at lower pressures, where cracks may not be entirely closed, may be lessened.

The maximum confining pressure at which velocities were measured in this study was $600 \mathrm{MPa}$ (Table 2 ). Figure 8 is a $V_{p}$-density plot of these data. Lines of constant mean atomic weight were corrected to $600 \mathrm{MPa}$ following the method of Birch (1961) outlined in Table 4. The seven diabase samples fall just below, and follow the slope of, the $\mathrm{m}=$ 22 line, which Birch reported as the mean atomic weight of common 


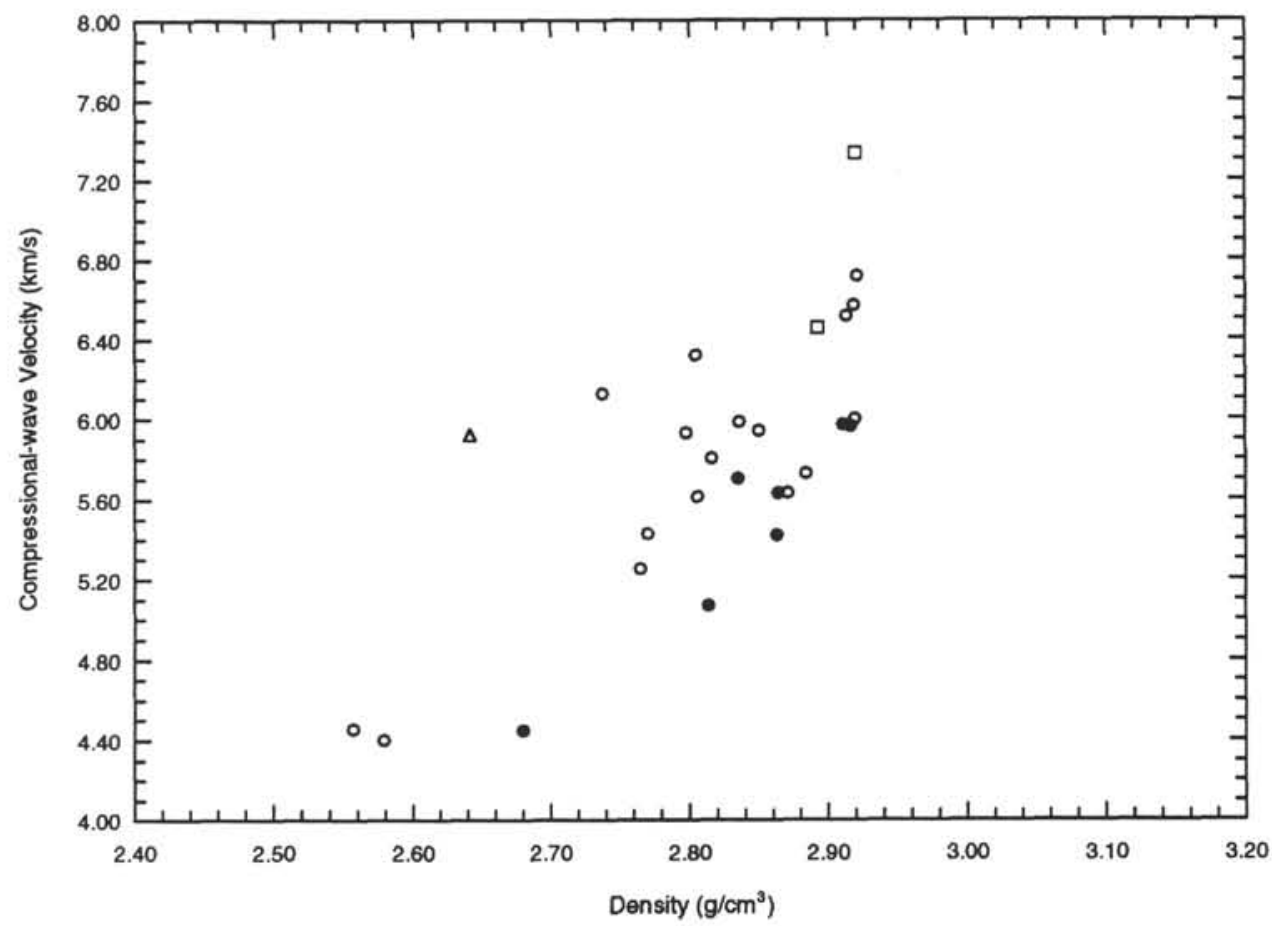

Figure 6. Velocity-density relationships for all Leg 139 samples, measured at $100 \mathrm{MPa}$ to minimize the effect of crack porosity. Squares are Hole 856A samples (2), circles represent Hole 857C/857D samples (24), and the triangle represents the single Hole 858G sample. Site 857 Purdue data, shown in Figure 8 at 600 MPa, are plotted as filled circles.

mafic igneous rocks. We believe that our data can be interpreted in terms of gross abundances of calcium, iron, and titanium since the effect of cracks on velocities is small to nonexistent at $600 \mathrm{MPa}$. Shipboard geochemical and normative composition data for nearby samples (Shipboard Scientific Party, 1992b, tables 26 and 27) report that calcium-rich anorthite $(\mathrm{m}=21.40)$ and diopside $(\mathrm{m}=21.65)$ typically form more than $50 \%$ of the rocks. Iron is found in the oxides ilme- nite $(\mathrm{m}=30.35)$ and magnetite $(\mathrm{m}=33.08)$, and in secondary sulfide minerals, which average $3 \%-4 \%$ and $2 \%-3 \%$, respectively. Ilmenite, the predominant oxide, contributes titanium.

\section{Mineralogy and Alteration}

The metamorphic assemblages observed in Holes 857C, 857D, and $858 \mathrm{G}$ are typical of upper zeolite (wairakite-chlorite-prehnite) to greenschist (albite-epidote-chlorite-actinolite) facies, representing a temperature range of $50^{\circ}-300^{\circ} \mathrm{C}$ (Shipboard Scientific Party, 1992b and 1992c). The original mineralogies of the medium- to coarsegrained Site 857 diabases, averaging $44: 43$ plagioclase/clinopyroxene with $3 \%$ oxide minerals and $10 \%$ mesostasis, have been replaced by chlorite, epidote, actinolite, and sulfides. The resultant rocks average 37:34 plagioclase/clinopyroxene with $3 \%$ oxides and less than $3 \%$ mesostasis, according to shipboard visual core descriptions and thin-section descriptions.

Correlation of oceanic rock velocities with low- to high-temperature alteration minerals is complicated by the often irregular nature of secondary mineral distribution (Christensen et al., 1989). However, we were able to quantify abundances of nonreflective constituents, interpreted as secondary minerals, in Site 857 diabases using computer-assisted image analysis (Table 3 ). No attempt was made to discriminate between various secondary minerals with the exception of sulfides in the absence of modal analyses and geochemical data; our goal was a first-order quantification of the percentages of those minerals that most affect velocity, density, and porosity values. Point counts were thus made of oxides (assumed to be primary constituents), sulfides and other secondary minerals.
Table 4. Parameters used to calculate the lines of constant mean atomic weight at maximum confining pressure of 600 MPa (Birch, 1961; Iturrino et al., 1991).

\begin{tabular}{ccccc}
\hline $\begin{array}{c}\text { Confining } \\
\text { pressure }\end{array}$ & $\begin{array}{c}\text { Average } \\
\text { MAW }\end{array}$ & $\mathrm{a}(\mathrm{km} / \mathrm{s})$ & $\mathrm{a}_{\mathrm{c}}(\mathrm{km} / \mathrm{s})$ & $\left.\mathrm{b}\left[(\mathrm{km} / \mathrm{s}) / \mathrm{g} / \mathrm{cm}^{3}\right)\right]$ \\
\hline \multirow{3}{*}{$600 \mathrm{MPa}$} & 20 & -1.423 & -1.513 & 3.169 \\
& 21 & -1.530 & -1.610 & 2.988 \\
& 22 & -2.123 & -2.213 & 2.929 \\
& 23 & -3.723 & -3.813 & 2.974 \\
& & & &
\end{tabular}

Note: These parameters were obtained with a least-squares solution of the form $V_{p}=\mathrm{a}+\rho \mathrm{b}$, where $V_{p}$ is in $\mathrm{km} / \mathrm{s}$ at $600 \mathrm{MPa}, \rho$ is in $\mathrm{g} / \mathrm{cm}^{3}$, $\mathrm{a}$ is the velocity intercept for velocities at $200 \mathrm{MPa}$ (Iturrino et al., 1991 ) and $1000 \mathrm{MPa}$ (Birch, 1961), $\mathrm{ac}_{\mathrm{c}}$ is the corrected intercept for velocities at $600 \mathrm{MPa}$, and $\mathrm{b}$ is the slope of the lines.

Examples of the occurrence and morphology of oxides and sulfides are shown in Plate 3. Fine-grained oxides are predominantly skeletal and rhomb-shaped (Pl. 3, Fig. 1; Sample 139-857D-20R-1, $79-81 \mathrm{~cm}$, and Fig. 2; Sample 139-857D-4R-1, 66-68 cm) and most ilmenite and magnetite grains are visibly altered. The alteration varies from fringes of a very fine-grained unidentified mineral at grain boundaries, which appears as a darker gray rim under reflected light in Plate 3, Figure 1, to complete replacement of magnetite by sphene (titanite), which often retains the original oxide morphology and is a mottled rich brown under transmitted light. Secondary and hydrothermal minerals, including sulfides, are present as static replacement products and as vein and fracture fillings in rocks from Holes $857 \mathrm{C}$, 857D, and $858 \mathrm{G} \mathrm{(Pl.} 1$ and 2). Pyrite is the most common sulfide and occurs in disseminated singular crystals (Pl. 3, Fig. 2) and elongated aggregates (PI. 3, Fig. 3; Sample 139-857D-21R-1, 68-70 cm). Pyrite with pyrrhotite overgrowths (PI. 3, Fig. 4; Sample 139-857D-9R-1, $51-52 \mathrm{~cm}$ ) is observed in some samples. The disseminated sulfides have a great variety of grain shapes, sizes and textures, and often display internal structures resembling pores (PI. 3, Fig. 4), that could 
contribute to microporosity. These characteristics suggest various emplacement histories. In contrast, the pyrite grains filling cracks and veins (Pl. 2) appear much fresher.

The velocity and density differences between adjacent Site 857 samples can be explained by the percentages of primary and secondary minerals in the rocks. The volume fraction of veins and cracks are also important, but their contribution to whole-minicore velocity is difficult to quantify in just two dimensions. Correlations of velocity and density with percent opaques (oxides + sulfides) and percent secondary minerals are shown in the four crossplots of Figure 9. The two low $V_{p}$-low $\rho$ outliers present in all four plots are the highly altered samples from the fine-grained chilled margin of Sample 139857D-1R-1 and were excluded from the curve fits. Positive correlations between $V_{p}-\%$ opaques and $\rho-\%$ opaques (Figs. $9 \mathrm{a}$ and $9 \mathrm{~b}$ ) reflect the dominant effect of secondary sulfides, mostly in the form of larger-grained pyrite crystals and aggregates $\left(V_{p} \cong 7.1 \mathrm{~km} / \mathrm{s}\right.$ (Gröschel-Becker et al., this volume, Site 856 type 5 sulfide); $\rho \cong$ $4.9-5.0 \mathrm{~g} / \mathrm{cm}^{3}$ ) over gine-grained disseminated primary oxides. Scatter in the data can be attributed to the occurrence of sulfides as macroscopic crack infill in some samples and as small disseminated grains in others. Partial to total replacement of magnitite $\left(V_{p} \cong 5.7-6.8\right.$ $\left.\mathrm{km} / \mathrm{s} ; \rho \cong 4.9-5.2 \mathrm{~g} / \mathrm{cm}^{3}\right)$ (Birch, $\left.1960 ; 1961\right)$ by sphene $(\rho \cong 3.4-3.5$ $\mathrm{g} / \mathrm{cm}^{3}$ ) lowers densities significantly and reduces velocities.

A slight decrease in both $V_{p}$ and density with increasing percentage of secondary minerals is shown in Figures $9 \mathrm{c}$ and $9 \mathrm{~d}$. The correlations are weak for tow principal reasons. Some secondary minerals increase velocities whereas others reduce velocities and can effectively cancel each other out if present in significant amounts. Their effects on densities are also variable. For example, chlorite $\left(V_{p} \cong 6.0 \mathrm{~km} / \mathrm{s} ; \rho \cong 2.8-3.2\right.$ $\left.\mathrm{g} / \mathrm{cm}^{3}\right)$, replacing plagioclase $\left(V_{p-A n 56} \cong 6.6 \mathrm{~km} / \mathrm{s} ; \rho_{A n 56} \cong 2.7 \mathrm{~g} / \mathrm{cm}^{3}\right)$ and some clinopyroxene $\left(V_{p \text {-Augite }} \cong 7.2 \mathrm{~km} / \mathrm{s} ; \rho \cong 3.3 \mathrm{~g} / \mathrm{cm}^{3}\right)$, can reduce velocities significantly but not change densities appreciably, whereas epidote $\left(V_{p} \cong 7.0-7.4 \mathrm{~km} / \mathrm{s} ; \rho \cong 3.3-3.6 \mathrm{~g} / \mathrm{cm}^{3}\right)$ and actinolite $\left(V_{p} \cong 7.0\right.$ (?) $\mathrm{km} / \mathrm{s} ; \rho \cong 3.2 \mathrm{~g} / \mathrm{cm}^{3}$ ) replacement increases velocities and may increase densities (Christensen and Wilkens, 1982; Christensen et al., 1989). Since secondary minerals were not individually identified in our petrographic analyses and recorded in the single category of nonreflective material, it was not possible to assess the contributions of fast and slow or high-density and low-density components.

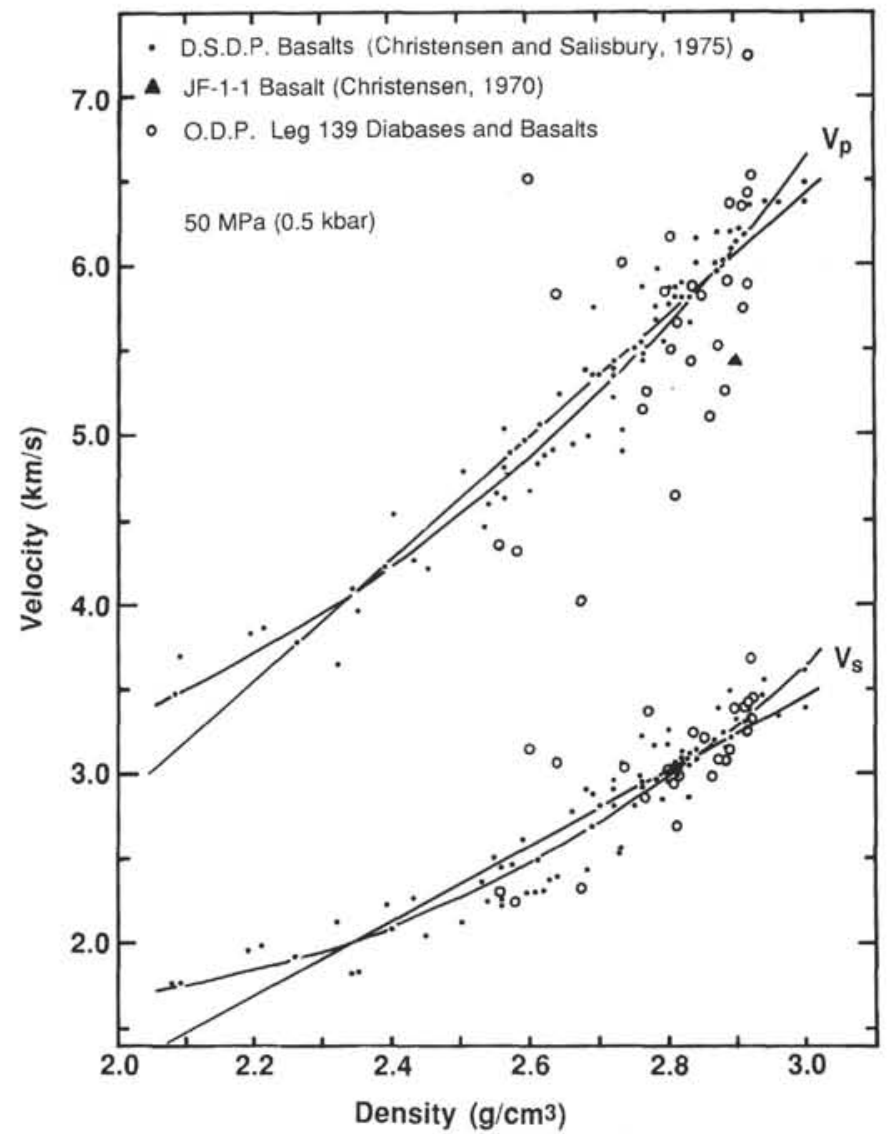

Figure 7. Comparison of Leg 139 data (open circles) with velocity-density systematics for DSDP basalts from the Atlantic and Pacific Oceans (dots) at $50 \mathrm{MPa}$ (after Christensen and Salisbury, 1975). Linear and nonlinear regression solutions are for DSDP data only. A dredged basalt from the Juan de Fuca Ridge (Christensen, 1970) is shown by the solid triangle.

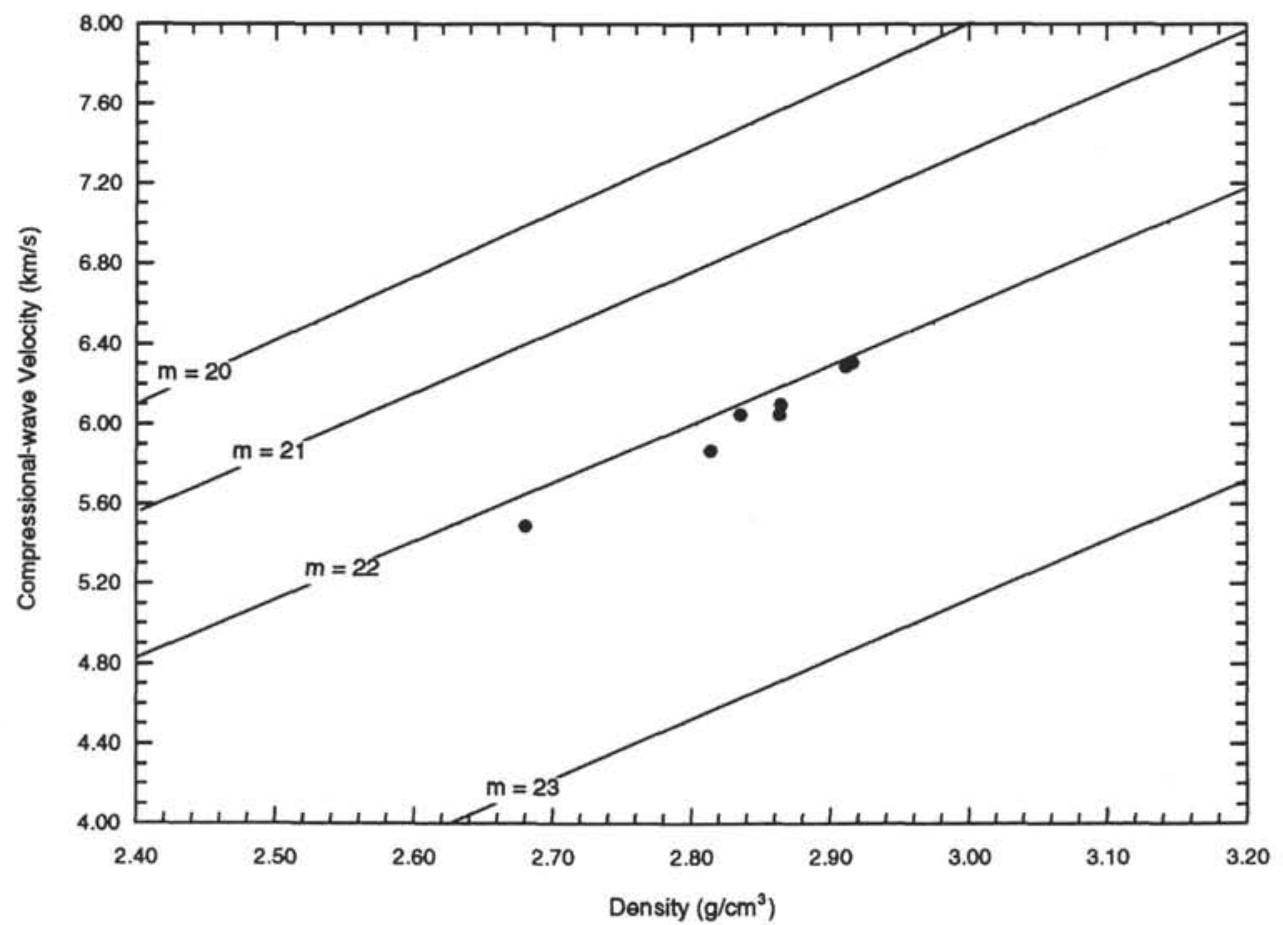

Figure 8. Velocity-density relationships for Site 857 samples measured at $600 \mathrm{MPa}$ at Purdue. Diagonal lines represent lines of constant mean atomic weight based on Birch's law. 

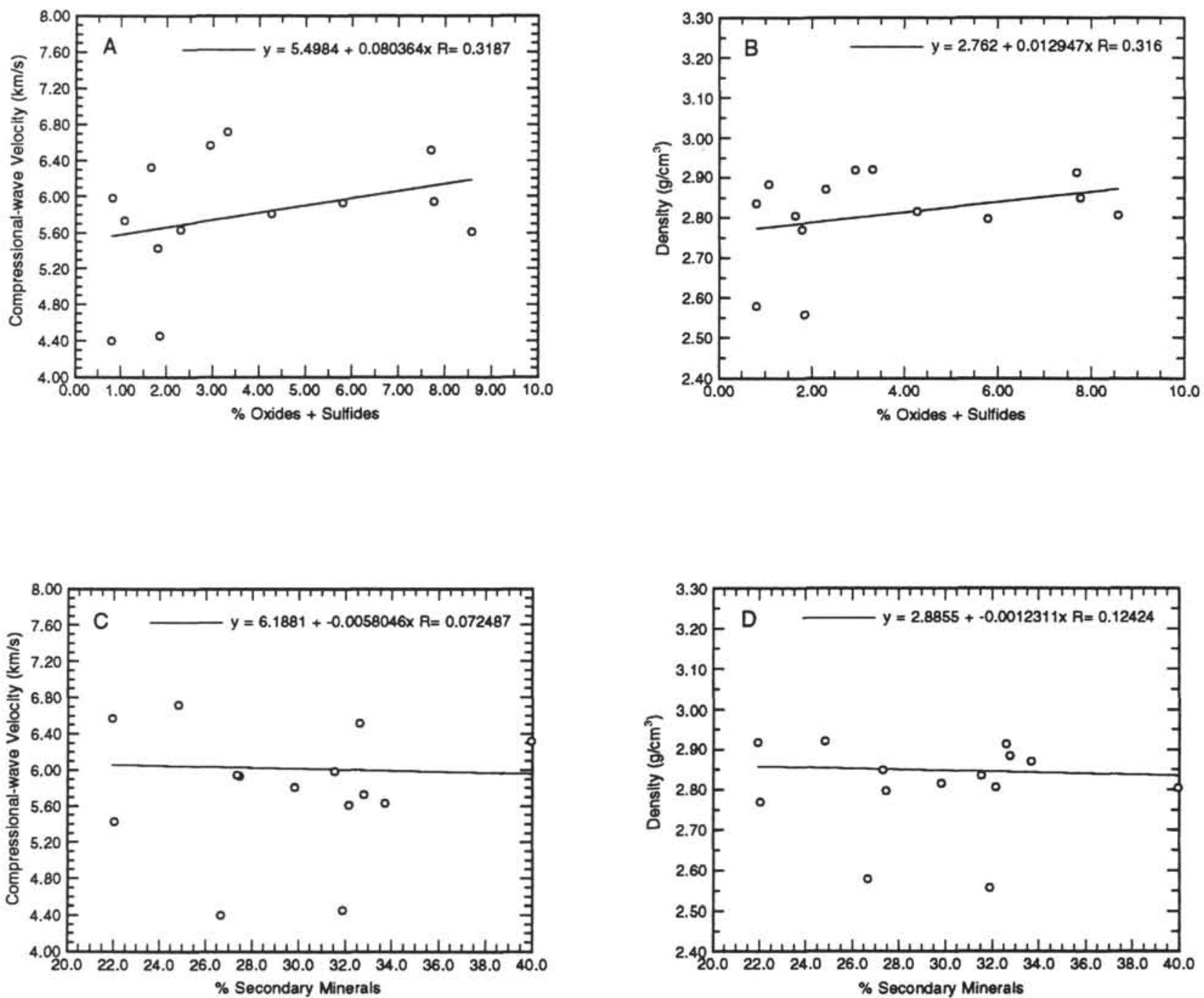

Figure 9. Cross-plots of $V_{p}$ (at $100 \mathrm{MPa}$ ) and density data for Hole 857D samples with point count data obtained using computer-assisted image analysis (Table 3). A and B show the effect on $V_{p}$ and density of the percent opaques (oxides + sulfides) identified in the samples; C and D are $V_{p}$ and density vs. the percentage of secondary minerals counted. Solid lines are best fit curves.

\section{CONCLUSIONS}

$V_{p}, V_{s}$, density, and porosity measurements for diabase and basalt samples from ODP Holes $856 \mathrm{~A}, 857 \mathrm{C}, 857 \mathrm{D}$, and $858 \mathrm{G}$ can be summarized as follows in terms of the acoustic structure of hydrothermal basement at sedimented spreading centers:

1. The mean compressional-wave velocity $(5.77 \mathrm{~km} / \mathrm{s}$ at $100 \mathrm{MPa}$; $5.65 \mathrm{~km} / \mathrm{s}$ at $50 \mathrm{MPa})$ and mean density $\left(2.81 \mathrm{~g} / \mathrm{cm}^{3}\right)$ of the Leg 139 diabases and basalts, subjected to low- to high-temperature hydrothermal alteration, agree with those measured for relatively fresh, unweathered MORB. This result suggests that different emplacement and alteration histories can produce mineral assemblages with similar physical properties.

2. Site 857 diabase sills are characterized by low $V_{p}$, low bulk density, and high porosities at the more highly altered margins, and by high $V_{p}$, high bulk density, and lower porosities at the generally less altered interiors.

3. The mean porosity of 17 Site 857 diabases is $7.26 \%$. Values range from $3.96 \%$ to $14.38 \%$, well above the average of $2 \%$ reported for extrusive Layer 2 basalts from Hole 504B. Microcracks formed in response to drilling and pressure relief can reduce velocities at low confining pressures. Thin-section observations show that partially filled cracks, veins, and intergranular pores inherent to the rocks also contribute to the overall high porosity values.

4. The physical properties of Leg 139 igneous rock samples are variable on a scale of millimeters to centimeters, as revealed in disparities between adjacent samples. The variability results primarily from the presence of cracks, veins, and a suite of low- to high-temperature hydrothermal alteration products. Textural and compositional changes in primary minerals are of secondary importance.

5 . Velocity differences in adjacent horizontally and vertically oriented minicores are not a result of $V_{p}$ anisotropy, as suggested previously for shipboard cube samples (Gröschel-Becker et al., 1992; Shipboard Scientific Party, 1992b). No evidence for preferred mineral or microcrack orientations was observed in thin sections and differences are mostly due to heterogeneity between samples as described above. Vertical shipboard velocities below $560.1 \mathrm{mbsf}$ may appear faster and horizontal velocities slower owing to the opening of numerous subvertical cracks as the cores were brought to atmospheric pressure conditions.

6. $V_{p}$ and densities for Site 857 diabases can be correlated with the percentages of oxide and sulfide minerals identified by computerassisted image analysis methods. The correlation with percentage of secondary minerals is weak. The relationship between these parameters is complex since different secondary minerals can reduce or increase velocities and densities if present in significant amounts. 


\section{ACKNOWLEDGMENTS}

HGB thanks JOI/USSAC for post-cruise funding. G. Eberli and J. Natland graciously allowed use of their laboratories and gave thoughtful comments on the manuscript, as did C. Harrison, B. Rosendahl, D. Fountain, and an anonymous reviewer. J. Wu performed index properties measurements at ODP/TAMU under the guidance of A. Fisher. NIC thanks the Office of Naval Research (Contract No. N00014-89-J1209) for its financial support.

\section{REFERENCES ${ }^{*}$}

Alt, J.C., Honnorez, J., Laverne, C., and Emmermann, R., 1986. Hydrothermal alteration of a $1 \mathrm{~km}$ section through the upper oceanic crust, Deep Sea Drilling Project Hole 504B: mineralogy, chemistry, and evolution of seawater-basalt interactions. J. Geophys. Res., 91:10309-10335.

Anderson, R.N., Honnorez, J., Becker, K., Adamson, A.C., Alt, J.C., Emmermann, R., Kempton, P.D., Kinoshita, H., Laverne, C., Mottl, M.J., and Newmark, R.L., 1982. DSDP Hole 504B, the first reference section over $1 \mathrm{~km}$ through Layer 2 of the oceanic crust. Nature, 300:589-594.

Becker, K., Sakai, H., Adamson, A.C., Alexandrovich, J., Alt, J.C., Anderson, R.N., Bideau, D., Gable, R., Herzig, P.M., Houghton, S.D., Ishizuka, H., Kawahata, H., Kinoshita, H., Langseth, M.G., Lovell, M.A., Malpas, J., Masuda, H., Merrill, R.B., Morin, R.H., Mottl, M.J., Pariso, J.E., Pezard, P.A.,Phillips, J.D., Sparks, J.W., and Uhlig, S., 1989. Drilling deep into young oceanic crust at Hole 504B, Costa Rica Rift. Rev. Geophys., 27:79-102.

Birch, F., 1960. The velocity of compressional waves in rocks to 10 kilobars, 1. J. Geophys. Res., 65:1083-1102.

1961. The velocity of compressional waves in rocks to 10 kilobars, 2. J. Geophys. Res., 66:2199-2224.

Carlson, R.L., and Herrick, C.N., 1990. Densities and porosities in the oceanic crust and their variations with depth and age. J. Geophys. Res., 95:91539170.

Christensen, N.I., 1970. Compressional wave velocities for basalts from the Juan de Fuca Ridge. J. Geophys. Res., 75:2773-2775.

1972. Compressional and shear wave velocities at 10 kilobars for basalts from the East Pacific rise. Geophys. J.R. Astron. Soc., 28:425-429.

1985. Measurements of dynamic properties of rocks at elevated temperatures and pressures. In Pincus, H.J., and Hoskins, E.R. (Eds.), Measurements of Rock Properties at Elevated Pressures and Temperatures: Philadelphia (Am. Soc. for Testing and Materials), ASTM STP 869:93-107.

Christensen, N.I., Blair, S.C., Wilkens, R.H., and Salisbury, M.H., 1979 Compressional wave velocities, densities and porosities of basalts from Holes 417A, 417D and 418A, Deep Sea Drilling Project Legs 51-53. In Donnelly, T., Francheteau, J., Bryan, W., Robinson, P., Flower, M., Salisbury, M., et al., Init. Repts. DSDP, 51, 52, 53 (Pt. 2): Washington (U.S. Govt. Printing Office), 1467-1471

Christensen, N.I., and Salisbury, M.H., 1975. Structure and constitution of the lower oceanic crust. Rev. Geophys. Space Phys., 13:57-86.

Christensen, N.I., Wepfer, W.W., and Baud, R.D., 1989. Seismic properties of sheeted dikes from Hole 504B, ODP Leg 111. In Becker, K., Sakai, H., et al., Proc. ODP, Sci. Results, 111: College Station, TX (Ocean Drilling Program), 171-174.

Christensen, N.I., and Wilkens, R.H., 1982. Seismic properties, density and composition of the Icelandic crust near Reydarfjordur. J. Geophys. Res., 87:6389-6395.

Curray, J.R., Moore, D.G., et al., 1982. Init. Repts. DSDP, 64 (Pts. 1 and 2): Washington (U.S. Govt. Printing Office).

Davis, E.E., Mottl, M.J., Fisher, A.T., et al., 1992.Proc. ODP, Init. Repts., 139: College Station, TX (Ocean Drilling Program).

Davis, E.E., and Villinger, H., 1992. Tectonic and thermal structure of the Middle Valley sedimented rift, northern Juan de Fuca Ridge. In Davis, E.E., Mottl, M.J., Fisher, A.T., et al., Proc. ODP, Init. Repts., 139: College Station, TX (Ocean Drilling Program), 9-41.

Einsele, G., Gieskes, J.M., Curray, J., Moore, D.M., Aguago, E., Aubry, M.P., Fornari, D., Guerrero, J., Kastner, M., Kelts, K., Lyle, M., Matoba, Y., MolinaCruz, A., Niemitz, J., Rueda, J., Saunders, A., Schrader, H., Simoneit, B. and Vacquier, V., 1980. Intrusion of basaltic sills into highly porous sediments and resulting hydrothermal activity. Nature, 283:441-445.

Fox, P.J., Schreiber, E., and Peterson, J.J., 1973. The geology of the oceanic crust: compressional wave velocities of oceanic rocks. J. Geophys. Res. 78:5155-5172.
Gröschel-Becker, H.M., Iturrino, G.J., Anselmetti, F.S., and Eberli, G.P., 1992. Seismic velocities and anisotropy of sulfide, diabase, basalt: ODP Leg 139 Sites 856, 857 and 858, N. Juan de Fuca Ridge. Eos, 73:500.

Hyndman, R.D., and Drury, M.J., 1976. The physical properties of oceanic basement rocks from deep drilling on the Mid-Atlantic Ridge at $23^{\circ} \mathrm{N} . J$. Geophys. Res., 81:4042-4052.

Ingle, J.C., Jr., Suyehiro, K., von Breymann, M.T., et al., 1990. Proc. ODP, Init. Repts., 128: College Station, TX (Ocean Drilling Program).

Iturrino, G.J., Christensen, N.I., Kirby, S., and Salisbury, M.H., 1991. Seismic velocities and elastic properties of oceanic gabbroic rocks from Hole 735B. In Von Herzen, R.P., Robinson, P.T., et al., Proc. ODP, Sci. Results, 118: College Station, TX (Ocean Drilling Program), 227-244.

Karson, J.A., and Rona, P.A., 1990. Block-tilting, transfer faults, and structural control of magmatic and hydrothermal processes in the TAG area, MidAtlantic Ridge $26^{\circ} \mathrm{N}$. Geol. Soc. Am. Bull., 102:1635-1645.

Langseth, M., Becker, K., Fisher, A., Davis, E., and the Leg 139 Scientific Party, 1992. Basement structure in Middle Valley and its relation to hydrothermal venting: Results from downhole logging on Leg 139. Eos, 73:520.

Lonsdale, P., and Becker, K., 1985. Hydrothermal plumes, hot springs, and conductive heat flow in the southern trough of Guaymas Basin. Earth Planet. Sci. Lett., 73:211-225.

Purdy, G.M., 1987. New observations of the shallow seismic structure of young oceanic crust. J. Geophys. Res., 92:9351-9362.

Rohr, K., and Gröschel-Becker, H., 1992. Correlation of drilling, well log and seismic reflection data over hydrothermal vents, ODP Leg 139, Middle Valley, Juan de Fuca Ridge. Eos, 73:500.

Rona, P.A., Denlinger, R.P., Fisk, M.R., Howard, K.J., Taghon, G.L., Klitgord, K.D., McClain, J.S., McMurray, G.R., and Wiltshire, J.C., 1990. Major off- axis hydrothermal activity on the northern Gorda Ridge. Geology, 18:493-496.

Rona, P.A., Thompson, G., Mottl, M.J., Karson, J.A., Jenkins, W.J., Graham, D., Mallette, M., Von Damm, K., and Edmund, J.M., 1984. Hydrothermal activity at the Trans-Atlantic Geotraverse Hydrothermal Field, Mid-Atlantic Ridge crest at $26^{\circ}$ N. J. Geophys. Res., 89:11365-11377.

Shipboard Scientific Party, 1992a. Site 856. In Davis, E.E., Mottl, M.J., Fisher, A.T., et al., Proc. ODP, Init. Repts., 139: College Station, TX (Ocean Drilling Program), 161-281.

1992b. Site 857. In Davis, E.E., Mottl, M.J., Fisher, A.T., et al., Proc. ODP, Init. Repts., 139: College Station, TX (Ocean Drilling Program), 283-429.

, 1992c. Site 858. In Davis, E.E., Mottl, M.J., Fisher, A.T., et al, Proc. ODP, Init. Repts., 139: College Station, TX (Ocean Drilling Program), 431-569.

Spudich, P., and Orcutt, J., 1980. Petrology and porosity of an oceanic crustal site: results from wave form modeling of seismic refraction data. $J$. Geophys. Res., 85:1409-1433.

Stephen, R.A., 1985. Seismic anisotropy in the upper oceanic crust. J. Geophys. Res., 90:11383-11396.

Tamaki, K., Pisciotto, K., Allan, J., et al., 1990. Proc. ODP, Init. Repts., 127: College Station, TX (Ocean Drilling Program).

Tamaki, K., Suyehiro, K., Allan, J., Ingle, J.C., Jr., and Pisciotto, K.A., 1992. Tectonic synthesis and implications of Japan Sea ODP drilling. In Tamaki, K., Suyehiro, K., Allan, J., McWilliams, M., et al., Proc. ODP, Sci. Results, 127/128 (Pt. 2): College Station, TX (Ocean Drilling Program), 1333-1348.

Wilkens, R.H., Christensen, N.I., and Slater, L., 1983. High-pressure seismic studies of Leg 69 and 70 basalts. In Cann, J.R., Langseth, M.G., Honnorez, J., Von Herzen, R.P., White, S.M., et al., Init. Repts. DSDP, 69: Washington (U.S. Govt. Printing Office), 683-686.

Wilkens, R.H., Fryer, G.J., and Karsten, J., 1991. Evolution of porosity and seismic structure of upper oceanic crust: importance of aspect ratios. $J$. Geophys. Res., 96:17891-17995.

* Abbreviations for names of organizations and publications in ODP reference lists follow the style given in Chemical Abstracts Service Source Index (published by American Chemical Society).

Date of initial receipt: 12 January 1993

Date of acceptance: 22 October 1993

Ms 139SR-248 

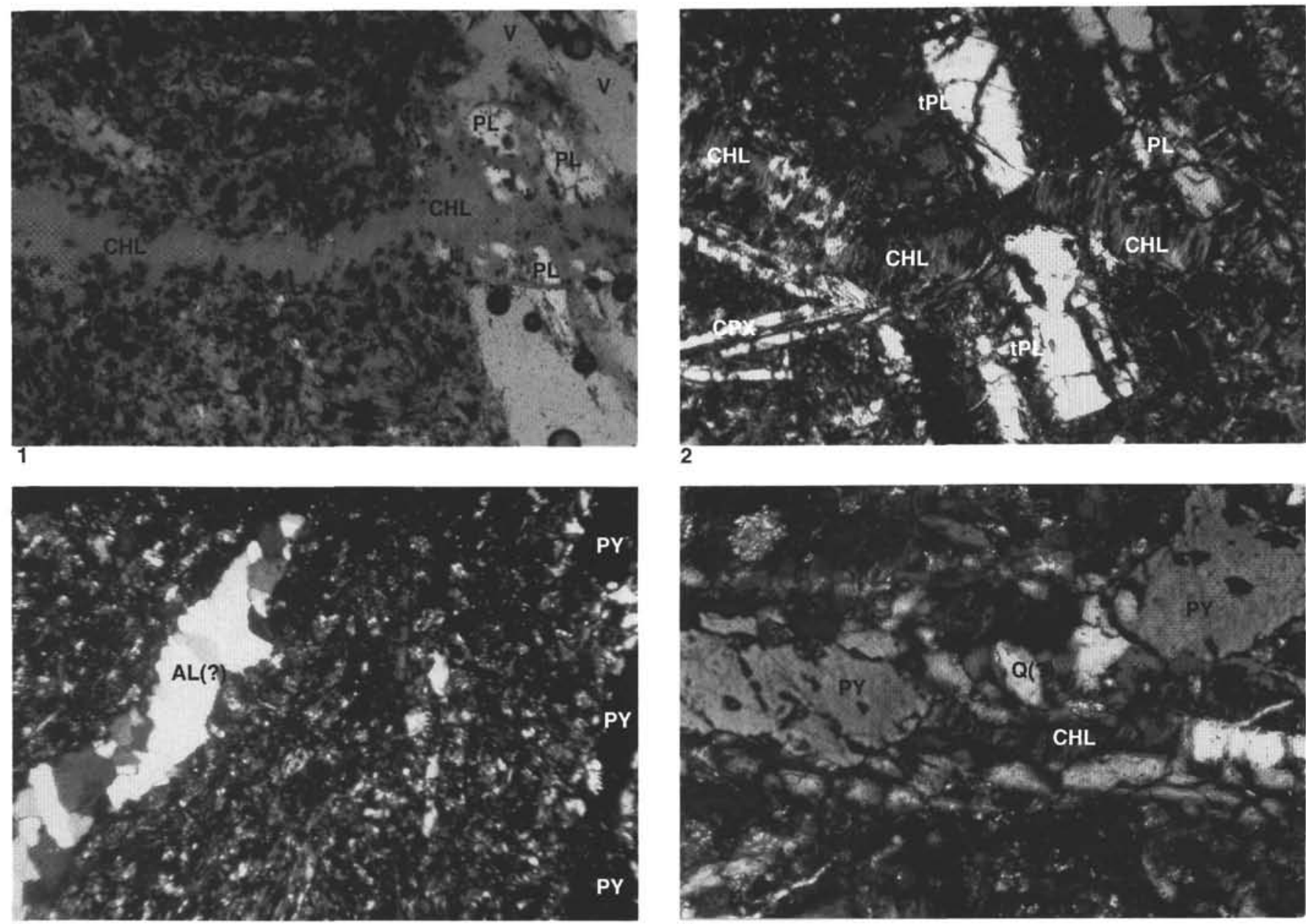

4

Plate 1. Thin-section photomicrographs of veins and microcracks in fine-grained samples from a chilled margin of a Hole 857D diabase sill. Field of view is 2.5 mm except for Fig. 4, for which it is $1 \mathrm{~mm}$. 1. Sample 139-857D-1R-1 (h), 24-26 cm. Subvertical vein (see text for orientation convention) filled with chlorite (CHL) intersects highly altered groundmass and fractured plagioclase (PL); infill extends into intergranular void (V) spaces (plane light). 2. Horizontal filled vein, in the same sample as in Fig. 1, cutting twinned plagioclase (tPL) and smaller laths. Fibrous chlorite blades clearly define width of vein (crossed nicols). 3. Sample 139-857D-1R-1 (v), 28-31 cm. Subvertical albite (AL)-filled (?) vein and formerly open microcrack partially rimmed with single pyrite (PY) crystals extends inward from edge of sample (top). Three pyrite grains are visible as black rhombs at right edge of photo (crossed nicols). 4. Enlarged view of the two pyrite crystals in the upper right corner of Fig. 3. Some small voids remain, but the crack aperture connecting pyrite grains is mostly filled with chlorite and rimmed with quartz (?) (Q) (combination transmitted/reflected light, crossed nicols). 

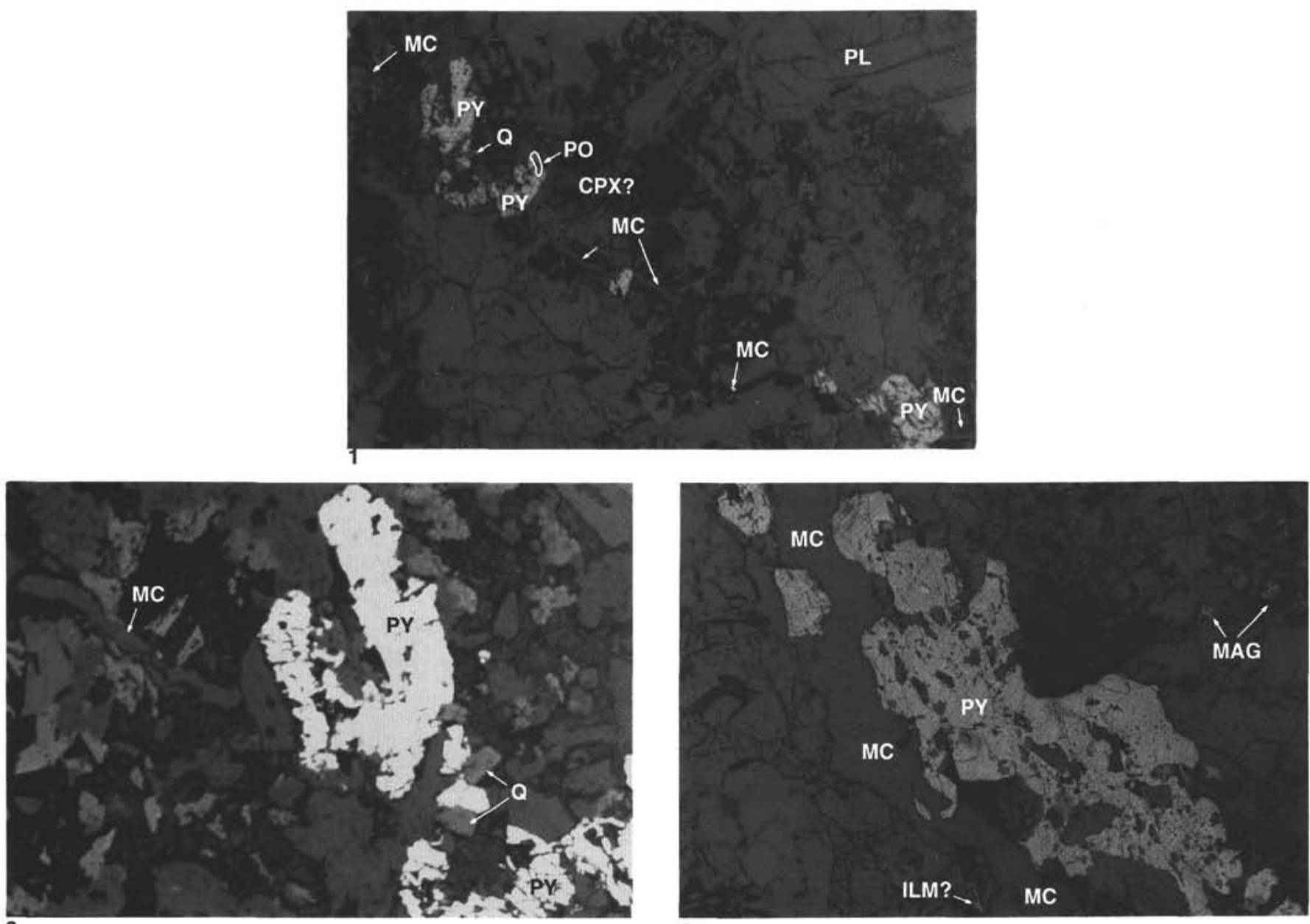

3

Plate 2. Thin-section photomicrographs of microfractures partially filled with sulfide crystals in less-altered diabase samples from Hole 857D. Field of view is $2.5 \mathrm{~mm}$ for Figs. 1 and 3 and $1 \mathrm{~mm}$ for Fig. 2. 1. Sample 139-857D-9R-1 (v), 54-57 cm. Open microcrack (MC) in slightly altered fine-grained diabase along which singular and multiple pyrite (PY), with possible minor pyrrhotite (PO), have crystallized. Minor quartz (Q) also is present. Note fractured and altered appearance of plagioclase (PL) and clinopyroxene (CPX) (reflected light). 2. Enlarged view of upper left corner of Fig. 1, showing patchy nature of secondary mineral recrystallization of primary minerals (reflected light). 3. Sample 139-857D-33R-1 (h), 37-39 cm. Major subhorizontal open microcrack with macroscopic altered pyrite, with intergranular filled voids, occurring as elongated packages of variously sized crystals. Note small skeletal magnetite (MAG) grains in upper right corner and rhomb-shaped ilmenite (?) (ILM) at bottom center of photo (reflected light). 


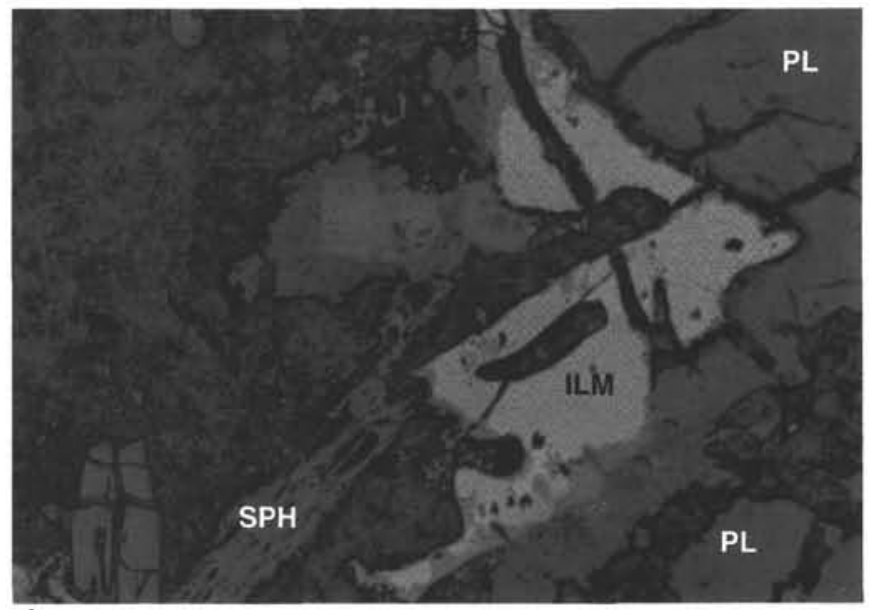

1

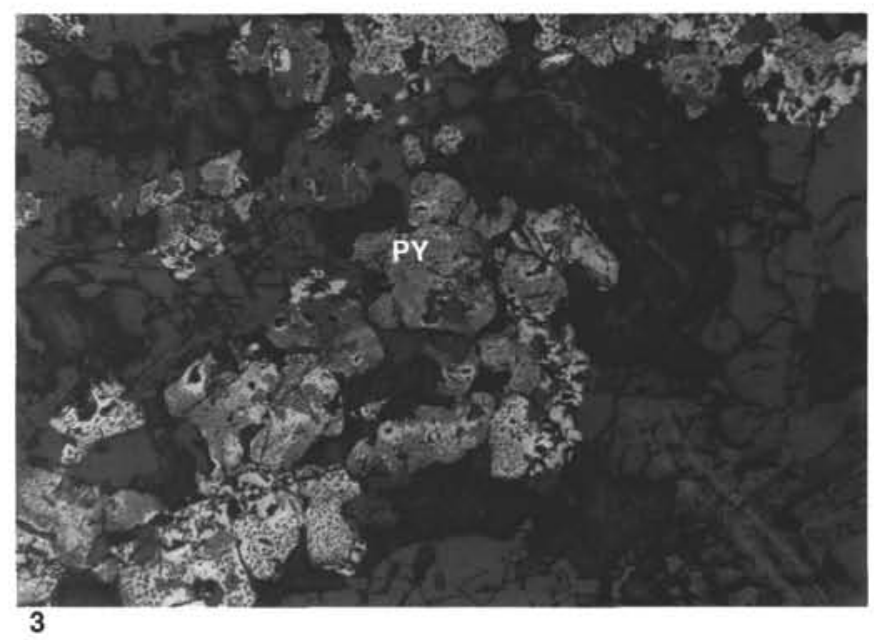

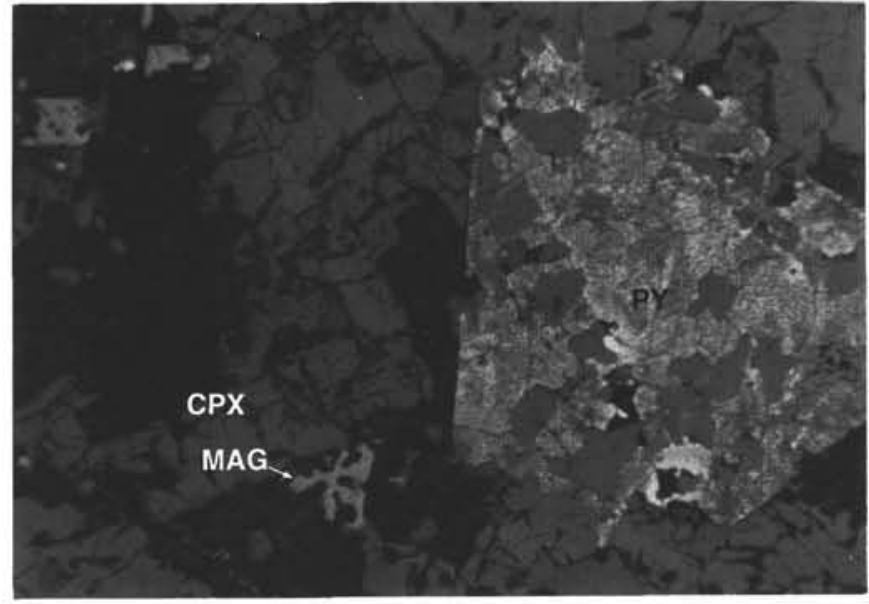

2

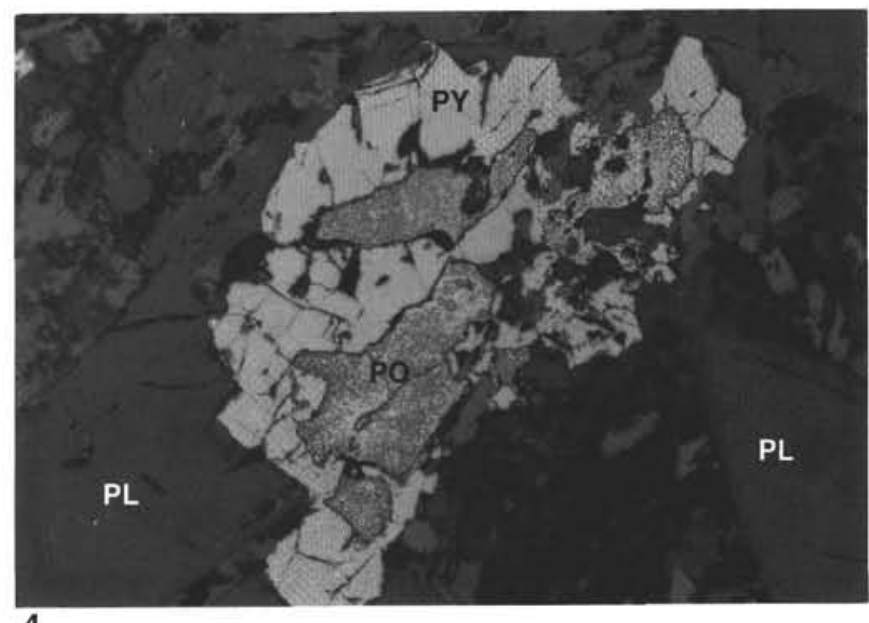

Plate 3. Thin-section photomicrographs of disseminated oxide and sulfide morphologies, and secondary mineral distribution, in moderately to highly altered diabase samples from Hole 857D. Field of view is $1 \mathrm{~mm}$ in Figs. 1 and 4 and $2.5 \mathrm{~mm}$ in Figs. 2 and 3. 1. Sample 139-857D-20R-1 (h), 79-81 cm. Subhedral ilmenite (ILM) with internal cracks and voids filled with dark gray, mottled secondary minerals; medium gray, irregular alteration rim is visible at grain boundaries. Sphene (SPH) replaces ilmenite in lower left and top center of photo. Plagioclase is the polished gray mineral to the right (reflected light). 2. Sample 139-857D-4R-1 (h), 66-68 cm. Macroscopic euhedral altered pyrite (PY), with possible minor pyrrhotite (PO), in clinopyroxene (CPX). Skeletal magnetite (MAG) and ilmenite laths are visible to the left of the photo (reflected light). 3. Sample 139-857D-21R-1 (v), 68-70 cm. Macroscopic aggregate of anhedral, rounded pyrite (PY) grains with complex internal structure occurs as megacryst in medium-grained diabase (reflected light). 4. Sample 139-857D-9R-1 (h), 51-53 cm. Fractured pyrite with pyrrhotite (PO) overgrowths observed in a fine-grained porphyritic diabase. Note eroded subhedral morphology and intergranular pores or vesicles in the pyrrhotite (reflected light). 